\title{
Modified Lomax Model: A heavy-tailed distribution for fitting large-scale real-world complex networks
}

\author{
Swarup Chattopadhyay ${ }^{1,3}$, Tanujit Chakraborty ${ }^{2 *}$, Kuntal Ghosh ${ }^{1}$, Asit K. das ${ }^{3}$ \\ ${ }^{1}$ Machine Intelligence Unit, Indian Statistical Institute Kolkata-700108, India \\ ${ }_{2} S Q C$ and OR, Indian Statistical Institute Kolkata-700108, India. \\ ${ }^{3}$ Department of C.S., Indian Institute of Engineering Science and Technology, Shibpur, Howrah, India.
}

\begin{abstract}
Real-world networks are generally claimed to be scale-free, meaning that the degree distributions follow the classical power-law, at least asymptotically. Yet, closer observation shows that the classical power-law distribution is often inadequate to meet the data characteristics due to the existence of a clearly identifiable non-linearity in the entire degree distribution in the log-log scale. The present paper proposes a new variant of the popular heavy-tailed Lomax distribution which we named as the Modified Lomax (MLM) distribution that can efficiently capture the crucial aspect of heavy-tailed behavior of the entire degree distribution of real-world complex networks. The proposed MLM model, derived from a hierarchical family of Lomax distributions, can efficiently fit the entire degree distribution of real-world networks without removing lower degree nodes as opposed to the classical power-law based fitting. The MLM distribution belongs to the maximum domain of attraction of the Frechet distribution and is right tail equivalent to Pareto distribution. Various statistical properties including characteristics of the maximum likelihood estimates and asymptotic distributions have also been derived for the proposed MLM model. Finally, the effectiveness of the proposed MLM model is demonstrated through rigorous experiments over fifty real-world complex networks from diverse applied domains.
\end{abstract}

Keywords: Complex networks; Degree distribution; Lomax distribution; Heavy-tailed distribution; Power-law; Statistical properties.

\section{Introduction}

The modeling and structural aspects of large scale real-world complex networks, including social, information, collaboration, communication, etc. have been well studied during the past decade [1, 2, 3, 4, 5] by many researchers. The World Wide Web, Twitter, Orkut, Youtube, DBLP, Wiki talk, Facebook, LinkedIn are examples of such large scale real-world complex networks. These networks are characterized by several important structural, emergent properties like degree distribution, correlation coefficient, average nearest neighbor, average path length, clustering coefficient, community structure, etc. Recently, the modeling and statistical aspects of such emergent structural properties, therefore, remain an important research area in the study of large scale real-world complex networks [6, 4, 7, 8, 9]. In this regard, the node degree distribution has been well studied and viewed as an important structural characteristic of real-world networks [10]. In 1999, Barabasi and Albert [11, 12] modeled the node degree distribution of the World Wide Web (WWW) using a power-law. Since then, many researchers have also favored the use of heavy tailed power-law in

* Corresponding author:

Email: tanujit_r@isical.ac.in (Tanujit Chakraborty)

Tel. +91-33-2575 3104/3100, Fax +91-33-2578-3357 
modeling the node degree distribution of real-world networks such as collaboration networks, communication networks, social networks, biological networks, etc [13, 14]. Mathematically, a quantity $x$ follows a power-law if it is drawn from a probability distribution $P(x) \propto x^{-\alpha}$ where, the parameter $\alpha$ is a positive constant and is known as exponent or scaling parameter of the distribution. Thus it is common to encounter the claim that most of the real-world networks are scale-free, meaning that the degree distributions follow single power-law. Despite this, a closer observation, while fitting, shows that the classical power-law distribution is often inadequate to meet the data characteristics adequately because of the existence of an identifiable non-linearity (bend) when the entire degree distribution is considered in log-log scale as shown in Figures $1 \mathrm{a}$ and $1 \mathrm{~b}$ (elaborated later).

This feature (non-linearity) of the entire degree distribution, depending on when and where it is considered or ignored, possibly constitute the reason why the universality vis-a-vis scarcity of scale-free networks has remained controversial ever since its inception 15, 16. The debate has continued to crop up time and again throughout the last twenty-one years [17, 18, 13, 14] and in very recent times too whence it has been claimed through an empirical and extensive study that the power-law distribution does not fit well in most cases and thereby produces a significant fitting error, followed by counter-claims [19].

This apart, researchers have also argued differently in favor of scale-free structure while suggesting some softer statistical criteria for scale-freeness [20, 21, 22]. Especially significant in this context is the following quote [22]: "The fact that heavy-tailed distributions occur in complex systems is certainly important (because it implies that extreme events occur more frequently than would otherwise be the case)... However, a statistically sound power-law is no evidence of universality without a concrete underlying theory to support it. Moreover, knowledge of whether or not a distribution is heavy-tailed is far more important than whether it can be fit using a power-law".

Several other heavy-tailed distributions such as lognormal, Pareto lognormal (PLN), double Pareto lognormal (DPLN), etc. also have been proposed in modeling the degree distribution of real-world networks instead of power-law [18, 23]. Recent research also recognized the deviations from a pure power-law distribution over various network data sets and recommended some other distributions for better modeling the heavy-tailed node degree distribution [21, 24, 25]. Thus, identifying the reasons for deviation of single power-law while fitting and looking for the alternative models which can efficiently capture the crucial aspect of heavy-tailed and long-tailed behaviour of the entire degree distribution of real-worlld complex networks continue to remain a challenging task of current research in the field of complexity science even as it steadily gravitates toward data science [20, 21, 22].

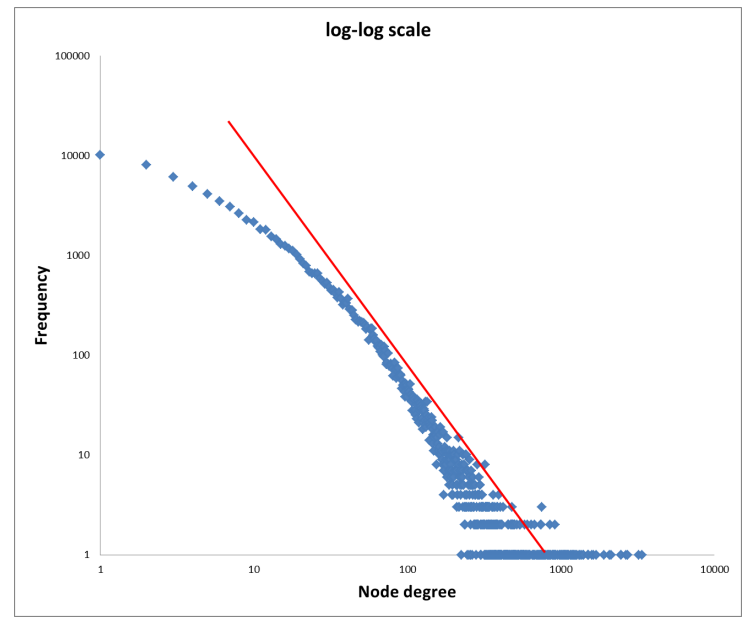

(a) Twitter network

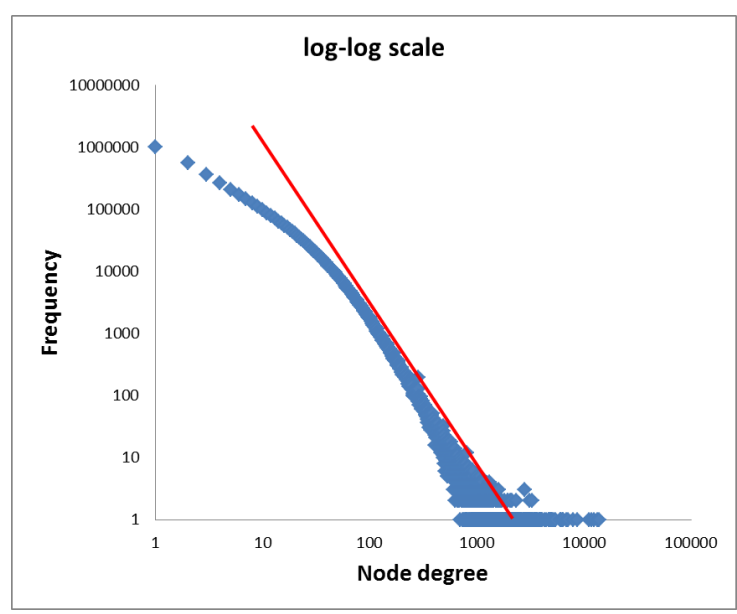

(b) LiveJournal network

Figure 1: Plot of degree distribution

Motivation: Networks are a powerful way to represent and study the structure of real-world complex 
systems. Across various applied domains of networks, it is common to encounter the claim that most of the real-world networks are scale-free, meaning that the degree distributions follow single power-law, though the universality of scale-free networks remains controversial as already discussed above.

Now consider an example where Figures $1 \mathrm{a}$ and $1 \mathrm{~b}$ that depict the plot of entire degree distribution in the log-log scale of the Twitter and LiveJournal social networks. The horizontal axis represents the unique degree value $(x)$, and the vertical axis represents the corresponding frequency. In these networks, a node represents a single user, and an edge represents a follower of that user. From these figures, it is clear that the pattern of the degree distribution of these networks does not match with the straight-line representation in the log-log scale through a single power-law. Usually, while fitting the node degree distribution, the single power-law is applied only for values of degree higher than some minimum (say, $x_{\min }$ ) and the exponent $\alpha$ is estimated from the data using MLE accordingly. Thus power-law distribution provides better fitting or in other words better inclined to the right tail of the data unless otherwise, some "unimportant" (i.e., lower degree) nodes are left out. Analytically, we can say that this inadequacy of fitting a single power-law occurs because of nonlinear behavior of the degree distribution curve in the log-log scale. This motivates the researchers to use other heavy-tailed probability models with non-negative exponent for better modeling the entire degree distribution of real-world networks. To capture these nonlinearities in the degree distribution of the real-world complex networks in a log-log scale, previous studies used various heavy-tailed probability distributions [21, 18, 24]. In this current research, we study the behavior of the entire degree distributions with a new variant of Lomax distribution that has wide applications in the field of actuarial science, reliability modeling, economics and computer science [26, 27, 28, 29]. The Lomax distribution is essentially a Pareto Type-II distribution that has been shifted so that its support begins at zero [27, 26]. Some extension and generalization of the Lomax distribution has been carried out for analyzing reliability and survival data sets in the past [27, 30, 31]. Recent research also focused on a new generalization of Pareto distribution with application to the breaking stress data [32]. This paper proposes a modified Lomax (MLM) distribution to be derived from a hierarchical family of Lomax distributions where the non-negative shape parameter is assumed to be expressible as a nonlinear function of the data.

Our contribution: The major contribution here is to develop a modified Lomax (MLM) distribution from a hierarchical family of Lomax distributions for efficient modeling of the entire degree distribution of real-world complex networks [27, 26]. The reasons for introducing MLM distribution is to provide greater flexibility and better fitting to the entire node degree distribution of complex networks compared to other popularly used heavy-tailed distributions. In other words, the proposed MLM model can be used for effective modeling the degree distribution of complex networks, coming from different disciplines, in the whole range of the data without discarding some of the lower degree nodes. Moreover, some statistical properties including extreme value and asymptotic behavior of the proposed MLM distribution have been studied in this context. We also provide mathematical arguments to explain the behavior of the likelihood surface for this nonlinear variant of the Lomax distribution, i.e., MLM distribution. A sufficient condition for the existence of the global maximum for the likelihood estimates is given using the notion of the coefficient of variations (CV) and discuss the parameter estimation procedures of the proposed MLM distribution. In order to justify the effectiveness of the proposed MLM distribution, we have compared it with the other common power-lawtype distributions, viz. power-law, Pareto, lognormal, exponential, power-law with exponential cutoff and Poisson [23, 13, 17]. The goodness-of-fit of the observed degree distribution is evaluated and compared using a few statistical measures, viz. bootstrap Chi-square, KL-divergence (KLD), mean absolute error (MAE) and root mean square error (RMSE). Several real-world complex networks from diverse fields have been used for experimental evaluation. Empirical results confirm the effectiveness of the proposed MLM distribution compared to other common distributions.

The remainder of the paper is organized as follows. Section 2 provides the details of the hierarchical family of Lomax distributions. We propose and interpret proposed modified Lomax (MLM) distribution in Section 3. Section 4 discusses the statistical properties, including extreme value and asymptotic behaviors of the proposed MLM distribution. Section 5 is devoted to the experimental results with a detailed analysis of the results over several real-world complex networks. Finally, Section 6 concludes the paper with a brief discussion. 


\section{Model}

In this section, we first introduce a new family of heavy-tailed Lomax (HLM) distributions. Further, we propose a relevant model from this newly introduced family to model the real-world heavy-tailed network data sets in the whole range.

\subsection{Genesis}

Lomax distribution has been used as an alternative to exponential, power-law, gamma and weibull distribution for modeling heavy tailed data sets [33, 34, 35, 36]. The cumulative distribution function $(\mathrm{CDF})$ and the probability density function (PDF) of the Lomax model are defined as follows:

Definition 1. A random variable $Z$ follows Lomax distribution with parameters $\alpha$ and $\sigma$ if the CDF is of the form:

$$
F(z)=1-\left(1+\frac{z}{\sigma}\right)^{-\alpha} ; z \geq 0
$$

where $\alpha(>0)$ is the shape parameter (real) and $\sigma(>0)$ is the scale parameter (real). The corresponding PDF is defined as follows:

$$
f(z)=\frac{\alpha}{\sigma}\left(1+\frac{z}{\sigma}\right)^{-\alpha-1} ; z \geq 0
$$

Below we introduce a new family of heavy tailed Lomax distributions which is right tail-equivalent to a power-law distribution.

Definition 2. A continuous random variable $X$ follows a family of heavy-tailed Lomax (HLM) distributions if and only if it has the following CDF:

$$
F(x)=1-(1+x)^{-m(x)} ; x \geq 0
$$

and $F(x)=0$ if $x \leq 0$, where $m:(0, \infty) \rightarrow \mathbb{R}^{+}$is a real, continuous, positive function which is differentiable on $(0, \infty)$ and satisfies the following conditions:

1. The function $m$ is strictly positive and have finite limit at infinity, i.e., $\lim _{x \rightarrow \infty} m(x)=\alpha(>0)$.

2. $\lim _{x \rightarrow 0^{+}}(1+x)^{m(x)}=1$ and $\lim _{x \rightarrow \infty}(1+x)^{m(x)}=\infty$.

3. $\frac{m^{\prime}(x)}{m(x)} \geq-\frac{1}{(1+x) \log (1+x)}, x>0$.

It can be easily verified that the CDF in (2) satisfying conditions (1), (2) and (3) is a genuine CDF which can also be expressed as follows:

$$
F(x)=1-\exp [-m(x) \log (1+x)], x>0
$$

The PDF of this new family of heavy-tailed Lomax distribution is of the form:

$$
f(x)=(1+x)^{-m(x)}\left[\frac{m(x)}{(1+x)}+m^{\prime}(x) \log (1+x)\right], \quad x>0 \quad \text { and } \quad f(x)=0, \quad x \leq 0 .
$$

There can be a wide variety of choices of $m(x)$ satisfying $\lim _{x \rightarrow \infty} m(x)=\alpha(>0)$. It is noted that the simplest choice of $m(x)=\alpha$ and $x=\frac{z}{\sigma}$ corresponds to the Lomax distribution. We further represent this newly introduced family of Lomax distributions as a hierarchical family in accordance with Pareto distribution [37. 
Definition 3. (HLM Type-I family of distributions) Supposed that a random variable $X$ folows HLM family of distributions as defined in (2). Then with a scale parameter $\sigma \in(0, \infty)$, the CDF of HLM Type-I family of distributions takes the following form:

$$
F(x)=1-\left[1+\left(\frac{x}{\sigma}-1\right)\right]^{-m\left(\frac{x}{\sigma}-1\right)}, \quad x>\sigma
$$

By taking $m\left(\frac{x}{\sigma}-1\right)=\alpha(>0)$, we obtain the classical Pareto Type-I distribution.

Definition 4. (HLM Type-II family of distribution) Supposed that a random variable $X$ folows HLM family of distributions as defined in (2). Then with a location parameter $\mu \in \mathbb{R}$ and a scale parameter $\sigma \in(0, \infty)$, the CDF of HLM Type-II family of distributions takes the following form:

$$
F(x)=1-\left(1+\frac{x-\mu}{\sigma}\right)^{-m\left(\frac{x-\mu}{\sigma}\right)}, \quad x>\mu
$$

By taking $m\left(\frac{x-\mu}{\sigma}\right)=\alpha(>0)$, we obtain the Pareto Type-II distribution. Also, in addition $\mu=0$ corresponds to the Lomax distribution.

Definition 5. (HLM Type-III family of distribution) Supposed that a random variable $X$ folows HLM family of distributions as defined in (2). Then with a location parameter $\mu \in \mathbb{R}$, scale parameters $\sigma \in(0, \infty)$ and a shape parameter $\gamma(>0)$, the CDF of HLM Type-III family of distributions takes the following form:

$$
F(x)=1-\left[1+\left(\frac{x-\mu}{\sigma}\right)^{\frac{1}{\gamma}}\right]^{-m\left[\left(\frac{x-\mu}{\sigma}\right)^{\frac{1}{\gamma}}\right]}, \quad x>\mu
$$

By taking $\left[m\left(\frac{x-\mu}{\sigma}\right)^{\frac{1}{\gamma}}\right]=1$, we obtain the Pareto Type-III distribution.

Obviously, the choice of $m(\cdot)$ function is subjective and any function $m$ satisfying conditions (1), (2) and (3) will give some known (unknown) heavy-tail Lomax distributions.

\section{Modified Lomax (MLM) Model}

The Lomax distribution does not provide great flexibility in modeling heavy-tailed data sets in the whole range similar to the power-law distribution. Due to this, the trend of parameter(s) induction to the baseline Lomax distribution has received increased attention in the recent years. Several generalized classes of distributions by adding additional parameters such as shape and or scale and or location in the distribution are available such as exponentiated Lomax (EL) 38], Beta-Lomax (BL) 39], exponential Lomax (ELomax) [40], Gamma-Lomax (GL) [1]] and Gumbel-Lomax (GuLx) model [42].

This paper provides a new modified version of the Lomax distribution called modified Lomax (MLM) distribution. MLM distribution is shown to be an asymmetric distribution, which provides great fit in modeling large-scale heavy-tailed data sets. The proposed MLM model is derived from the HLM family of distributions (in particular, HLM Type-II model) that can efficiently model the entire degree distribution of real-world networks. In other words, the proposed MLM model can be used for effective modeling the degree distribution of real-worlld complex networks in the whole range without discarding lower degree nodes. We define a relevant model from the newly introduced HLM Type-II family with the location parameter $\mu=0$ and we choose a flexible $m(\cdot)$ function that depends on two shape parameters $\alpha$ and $\beta$ satisfying $\lim _{x \rightarrow \infty} m(x)=\alpha$. The rational behind adding an additional shape parameter in the HLM Type-II family of distribution will make the statistical model more flexible, simple and have physical interpretation. This idea of generalization should suffice the practical needs of working with the non linear exponent to address the structural issue (degree distribution) of real-world complex networks. 
Now we choose a nonlinear function $m$ that adds a nonlinear exponents while fitting heavy-tailed HLM Type-II model in the degree distributions is as follows:

$$
m(x)=\alpha\left(\frac{\log (1+x)}{1+\log (1+x)}\right)^{\beta} .
$$

The chosen $m(x)$ approaches to $\alpha$ from below if $-1<\beta<0$ as $x \rightarrow \infty$ and approaches to $\alpha$ from above for $\beta>0$ as $x \rightarrow \infty$. Note that, the function $m(x)$ as defined above includes the constant function (in this case $\alpha$ ) as special cases by setting $\beta=0$. The derivative of $m(x)$ is given by

$$
m^{\prime}(x)=\frac{\alpha \beta}{x+1}\left(\frac{\log (1+x)}{1+\log (1+x)}\right)^{\beta-1} \cdot(1+\log (1+x))^{-2} .
$$

Now, we define a relevant model with the above choice of $m(\cdot)$ in the HLM Type-II model with $\mu=0$ and name it as Modified Lomax Model to be denoted by $\operatorname{MLM}(\alpha, \beta, \sigma)$. This modification to the Lomax distribution provides more flexibility in the data modeling since the non-negative shape parameter are assumed to be expressed as a nonlinear function of the empirical data. Thus the proposed MLM model with parameters $\alpha, \beta, \sigma$ could be useful for modeling the heavy-tailed degree distribution of real-world complex network data sets in the whole range.

Definition 6. (Modified Lomax Distribution) A continuous random variable $X$ follows $M L M(\alpha, \beta, \sigma)$ distribution with $\alpha(>0)$ and $\beta(>-1)$ as the shape parameters and $\sigma(>0)$ as the scale parameter if the $\mathrm{CDF}$ takes the following form:

$$
F(x)=1-\exp \left[-\alpha \frac{\log ^{\beta+1}(1+x / \sigma)}{[1+\log (1+x / \sigma)]^{\beta}}\right], x>0,
$$

and $F(x)=0$ if $x \leq 0$. The corresponding $\mathrm{PDF}$ is given by,

$$
f(x)=\frac{\alpha\left[\beta+1+\log \left(1+\frac{x}{\sigma}\right)\right]\left[\log \left(1+\frac{x}{\sigma}\right)\right]^{\beta}}{\sigma\left(1+\frac{x}{\sigma}\right)\left[1+\log \left(1+\frac{x}{\sigma}\right)\right]^{\beta+1}} \exp \left[-\alpha \frac{\left[\log \left(1+\frac{x}{\sigma}\right)\right]^{\beta+1}}{\left[1+\log \left(1+\frac{x}{\sigma}\right)\right]^{\beta}}\right], x>0
$$

and $f(x)=0$ if $x \leq 0$.

This MLM model includes Lomax distribution $\beta=0$ as particular case. In addition, it belongs to the new family of HLM Type-II distribution satisfying the condition: $\lim _{x \rightarrow \infty} m(x)=\alpha(>0)$. Due to the addition of an additional parameter $\beta$ in the exponents of the Lomax distribution generates various shapes (unimodal and bimodal) and provides greater flexibility (nonlinearity and heavy-tail) as shown in Figure 2. We study the monotonicity for the PDF of the proposed MLM model in Theorem 1 below.

Theorem 1. Let $X$ be the random variable follows $M L M(\alpha, \beta, \sigma)$ distribution, then the $\mathrm{PDF}$ as in (4) is a decreasing function for $-1<\beta<0$.

Proof. Differentiating (4) w.r.t. $x$, we have

$$
\begin{aligned}
f^{\prime}(x) & =-\frac{\alpha^{2}[1-F(x)]\left[\beta+1+\log \left(1+\frac{x}{\sigma}\right)\right]^{2}\left[\log \left(1+\frac{x}{\sigma}\right)\right]^{2 \beta}}{\sigma^{2}\left(1+\frac{x}{\sigma}\right)^{2}\left[1+\log \left(1+\frac{x}{\sigma}\right)\right]^{2 \beta+2}} \\
& -\frac{\alpha[1-F(x)]\left\{\left[\beta+1+\log \left(1+\frac{x}{\sigma}\right)\right]\left[\log \left(1+\frac{x}{\sigma}\right)\right]^{\beta}+(1+\beta)\left[\log \left(1+\frac{x}{\sigma}\right)\right]^{\beta-1}\right\}}{\sigma^{2}\left(1+\frac{x}{\sigma}\right)^{2}\left[1+\log \left(1+\frac{x}{\sigma}\right)\right]^{\beta+1}} \\
& +\frac{\alpha[1-F(x)](1+\beta)\left[\beta+1+\log \left(1+\frac{x}{\sigma}\right)\right]\left[\log \left(1+\frac{x}{\sigma}\right)\right]^{\beta-1}}{\sigma^{2}\left(1+\frac{x}{\sigma}\right)^{2}\left[1+\log \left(1+\frac{x}{\sigma}\right)\right]^{\beta+2}}
\end{aligned}
$$



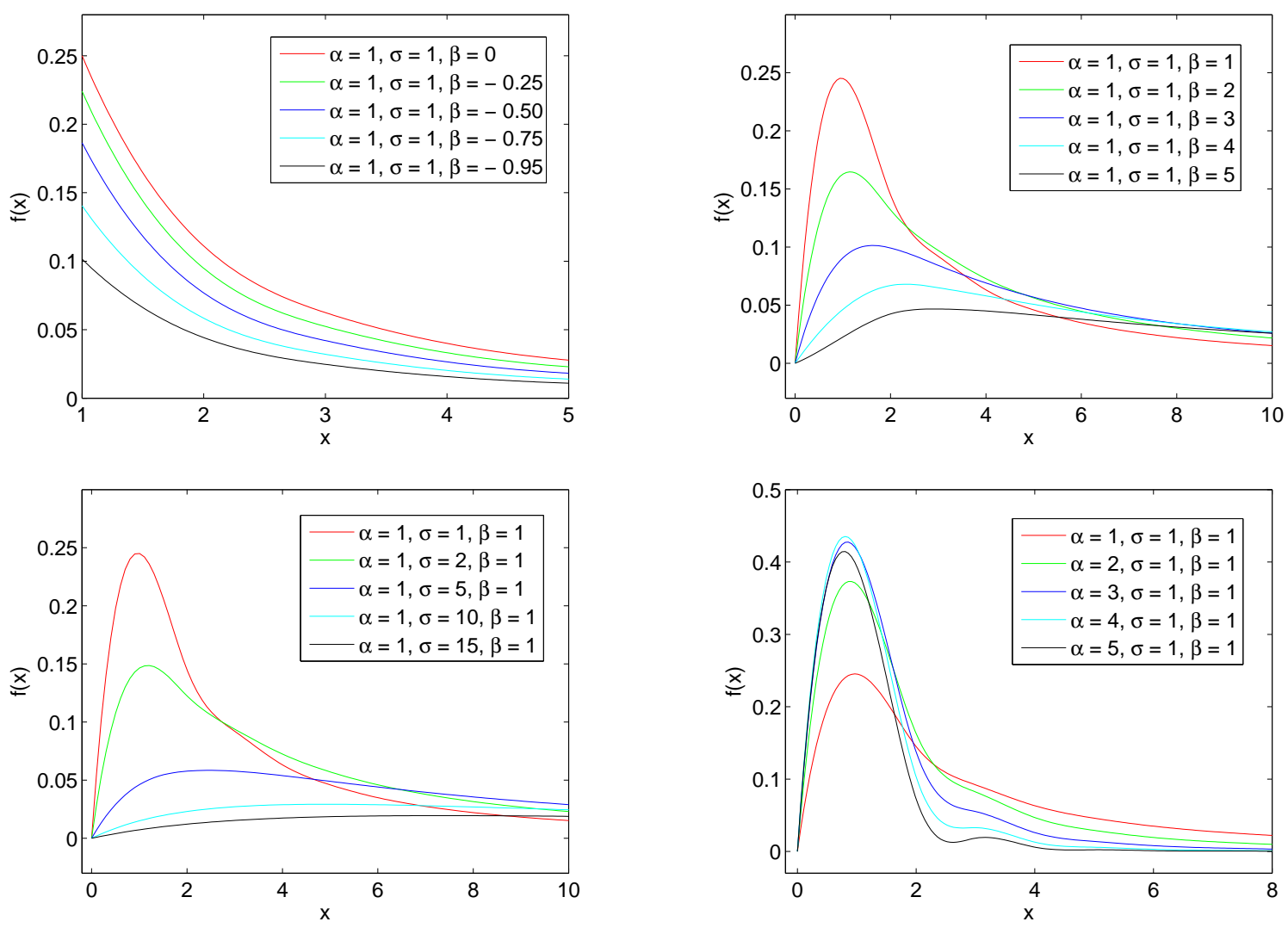

Figure 2: Plot of the PDFs of MLM distribution

Trivially, if $-1<\beta<0$, then $f^{\prime}(x)<0$. Thus, $f(x)$ is decreasing function if $\beta \in(-1,0)$.

\section{Statistical Properties of the MLM distribution}

\subsection{Characterization and existence of the likelihood}

Initially we characterize the maximum likelihood estimates (MLEs) of the parameters $\alpha$ and $\sigma$ of a Lomax distribution. Subsequently, we derived a sufficient condition for the existence of MLEs of the MLM distribution using coefficient of variation (CV). Given a set of samples $\left\{x_{i}\right\}$ of size $n$, the log-likelihood function for the Lomax distribution, after dividing it by the sample size $n$, is given by

$$
\ell(\alpha, \sigma)=\log \alpha-\log \sigma-\frac{(\alpha+1)}{n} \sum_{i=1}^{n} \log \left(1+\frac{x_{i}}{\sigma}\right)
$$

Differentiating (6) w.r.t. $\alpha$ and $\sigma$, respectively, we have:

$$
\begin{gathered}
\frac{\partial \ell(\alpha, \sigma)}{\partial \alpha}=\frac{1}{\alpha}-\frac{1}{n} \sum_{i=1}^{n} \log \left(1+\frac{x_{i}}{\sigma}\right) \\
\frac{\partial \ell(\alpha, \sigma)}{\partial \sigma}=-\frac{1}{\sigma}+\frac{(1+\alpha)}{n \sigma} \sum_{i=1}^{n}\left(\frac{x_{i}}{\sigma+x_{i}}\right)
\end{gathered}
$$


Equating to zero the derivative of $\ell(\alpha, \sigma)$ w.r.t. $\alpha$ in (7), we obtain $\hat{\alpha}=\alpha(\sigma)$ as follows:

$$
\hat{\alpha}=\alpha(\sigma)=\frac{n}{\sum_{i=1}^{n} \log \left(1+\frac{x_{i}}{\sigma}\right)}
$$

Differentiating (9) w.r.t. $\sigma$ we have,

$$
\alpha^{\prime}(\sigma)=\frac{\hat{\alpha}^{2}}{n \sigma} \sum_{i=1}^{n} \frac{x_{i}}{\sigma+x_{i}}
$$

It is important to note that there is no closed form solution to the likelihood based on (7) and (8), and a suitable numerical algorithm (for example, Newton-Raphson method) can be employed to obtain the maximum likelihood estimates (MLEs) of the $\alpha$ and $\sigma$. Different estimation procedures of the MLEs have been discussed in previous literature, for example see [43]. But for small or medium-sized samples, anomalous behavior of the likelihood surface can be encountered when sampling from the Lomax distribution. In this paper, we characterize the profile log-likelihood function in terms of the coefficient of variation $(\mathrm{CV})$, defined as follows:

Definition 7. The CV is the ratio of the standard deviation $(s)$ to the mean $(\mu)$,

$$
C V=\frac{s}{\mu}
$$

where $\mu=\frac{1}{n} \sum_{i=1}^{n} x_{i}$ and $s=\sqrt{\frac{1}{n} \sum_{i=1}^{n} x_{i}^{2}-\mu^{2}}$.

By using standard notation, the profile log-likelihood function based on equation 6, is given by

$$
\ell_{p}(\sigma)=\sup \ell(\hat{\alpha}, \sigma)=\log (\alpha(\sigma))-\log \sigma-1-\frac{1}{\alpha(\sigma)}
$$

Differentiating (11) w.r.t. $\sigma$, we have the following:

$$
\ell_{p}^{\prime}(\sigma)=\frac{\alpha^{\prime}(\sigma)}{\alpha(\sigma)}-\frac{1}{\sigma}+\frac{\alpha^{\prime}(\sigma)}{[\alpha(\sigma)]^{2}}
$$

Below we present the following lemmas which will be useful to find the sufficient condition for the existence for the global maximum of the profile log-likelihood function (11).

Lemma 1. The following limit holds:

1. $\lim _{\sigma \rightarrow \infty} \sigma \log \left(1+\frac{x}{\sigma}\right)=x$

2. $\lim _{\sigma \rightarrow \infty} \frac{\sigma x}{\sigma+x}=x$;

3. $\lim _{\sigma \rightarrow \infty} \sigma^{2}\left(\log \left(1+\frac{x}{\sigma}\right)-\frac{x}{\sigma+x}\right)=\frac{x^{2}}{2}$.

Proof. The proof is elementary and can easily be done using series expansions.

Lemma 2. The following limit holds:

1. $\lim _{\sigma \rightarrow \infty} \frac{1}{\alpha(\sigma)}=0$;

2. $\lim _{\sigma \rightarrow \infty} \frac{\alpha(\sigma)}{\sigma}=\frac{1}{\bar{x}}$, where $\bar{x}$ is the sample mean;

3. $\ell_{0} \equiv \lim _{\sigma \rightarrow \infty} \ell_{p}(\sigma)=\log \left(\frac{1}{\bar{x}}\right)-1$. 
Proof. The proofs are straightforward and can be done using Lemma (11).

1. $\lim _{\sigma \rightarrow \infty} \frac{1}{\alpha(\sigma)}=\lim _{\sigma \rightarrow \infty} \frac{1}{n} \sum_{i=1}^{n} \log \left(1+\frac{x_{i}}{\sigma}\right)=\lim _{\sigma \rightarrow \infty} O\left(\frac{1}{\sigma}\right)=0$.

2. $\lim _{\sigma \rightarrow \infty} \frac{\alpha(\sigma)}{\sigma}=\lim _{\sigma \rightarrow \infty} \frac{n}{\sigma \sum_{i=1}^{n} \log \left(1+\frac{x_{i}}{\sigma}\right)}=\frac{n}{\sum_{i=1}^{n} x_{i}}=\frac{1}{\bar{x}}$.

3. $\lim _{\sigma \rightarrow \infty} \ell_{p}(\sigma)=\lim _{\sigma \rightarrow \infty}\left[\log \left(\frac{\alpha(\sigma)}{\sigma}\right)-1-\frac{1}{\alpha(\sigma)}\right]=\log \left(\frac{1}{\bar{x}}\right)-1$.

A sufficient condition for monotonic increasing (decreasing) for the profile log-likelihood function is presented in Theorem (2) below, for sufficiently large $\sigma$. Also, we present a sufficient condition for the existence of global maximum corresponding to the likelihood function for the Lomax distribution to be at a finite point in Corollary (1).

Theorem 2. Let $X$ follows $\operatorname{LM}(\alpha, \sigma)$ distribution with $\alpha, \sigma>0$. A sufficient condition for $\ell_{p}(\sigma)$ to be monotonically decreasing function is $\mathrm{CV}>1$ for $\sigma \rightarrow \infty$, and if $\mathrm{CV}<1$, it is monotonically increasing.

Proof. Using (9) and (10) in Eqn. (12), we can write $\ell_{p}^{\prime}(\sigma)$ as:

$$
\ell_{p}^{\prime}(\sigma)=-\frac{1}{\sigma}\left[\frac{\sum_{i=1}^{n} \log \left(1+\frac{x_{i}}{\sigma}\right)-\sum_{i=1}^{n} \frac{x_{i}}{\sigma+x_{i}}}{\sum_{i=1}^{n} \log \left(1+\frac{x_{i}}{\sigma}\right)}\right]+\frac{1}{n \sigma} \sum_{i=1}^{n} \frac{x_{i}}{\sigma+x_{i}}
$$

Using the limits of Lemma (10) in Eqn. (13), we have

$$
-\lim _{\sigma \rightarrow \infty} \sigma^{2} \ell_{p}^{\prime}(\sigma)=\frac{1}{2} \times \frac{\sum_{i=1}^{n} x_{i}^{2}}{\sum_{i=1}^{n} x_{i}}-\bar{x} .
$$

Finally, we note that $-\lim _{\sigma \rightarrow \infty} \sigma^{2} \ell_{p}^{\prime}(\sigma)>0$ when the R.H.S of Eqn.(14) is strictly greater than 0. Alternatively, the likelihood function is monotonic decreasing when $\frac{1}{2 n} \sum_{i=1}^{n} x_{i}^{2}-\bar{x}^{2}>0$, or, equivalently, $\mathrm{CV}>1$. In a similar way, we can show that if $\mathrm{CV}<1$, then the $\ell_{p}(\sigma)$ is monotonic increasing function for sufficiently large $\sigma$.

Remark 1. As a consequence of Theorem (2), it can be immediately concluded that $\ell_{p}(\sigma)$ tends to $\ell_{0}$ based on Lemma (2) and $\ell_{p}(\sigma)$ is a monotonic function for sufficiently large $\sigma$. The value of CV as a measure that can be useful to determine when $\ell_{p}(\sigma)$ will be monotonic increasing or decreasing function for sufficiently large $\sigma$.

Corollary 1. Given a set of samples $\left\{x_{i}\right\}$ of $(+)$ ve numbers with CV $>1$, the profile likelihood function for the $\operatorname{LM}(\alpha, \sigma)$ distribution has a global maximum at a finite point.

Proof. For small or moderate values of $\sigma$, using (9), we have

$$
\lim _{\sigma \rightarrow 0} \alpha(\sigma)=\lim _{\sigma \rightarrow 0} \frac{n}{\sum_{i=1}^{n} \log \left(1+\frac{x_{i}}{\sigma}\right)}=0 .
$$

Now, using (15) in (11) we have the following:

$$
\lim _{\sigma \rightarrow 0} \ell_{p}(\sigma)=-\infty
$$

Since $\ell_{p}(\sigma)$ is a continuous and monotonic decreasing function for sufficiently large $\sigma$ (as in Theorem 21) and using (16), we can conclude that a global maximum exists at a finite point when $\mathrm{CV}>1$. 
Remark 2. Corollary 1 shows that the likelihood function for the Lomax distribution has a global maximum for the samples $\left\{x_{i}\right\}$ with $\mathrm{CV}>1$ at a finite point. The calculation of $\mathrm{CV}$ is completely based on available empirical data and easy to compute. The existence of MLE based on CV for the MLM distribution will also holds as because MLM model reduce to Lomax distribution when $\lim _{x \rightarrow \infty} m(x)=\alpha$. This can be empirically validated in section 55.3 and will be useful useful from practitioner's point of view.

\subsection{MLE of parameters}

In this section, the maximum likelihood estimates are derived for parameters $\alpha$, $\beta$, and $\sigma$ of MLM distribution. Let $x_{1}, x_{2}, \ldots, x_{n}$ be a sample of size $n$ from $\operatorname{MLM}(\alpha, \beta, \sigma)$ distribution. Then the log-likelihood function for the vector of parameters $\Theta=(\alpha, \beta, \sigma)^{T}$ is given by

$$
\begin{aligned}
\ell \equiv \ell(x ; \alpha, \beta, \sigma) & =n \log (\alpha)-\sum_{i=1}^{n} \log \left(\sigma+x_{i}\right)+\sum_{i=1}^{n} \log \left[\beta+1+\log \left(1+\frac{x_{i}}{\sigma}\right)\right] \\
& +\beta \sum_{i=1}^{n} \log \left[\log \left(1+\frac{x_{i}}{\sigma}\right)\right]-(\beta+1) \sum_{i=1}^{n} \log \left[1+\log \left(1+\frac{x_{i}}{\sigma}\right)\right] \\
& -\alpha \sum_{i=1}^{n} \frac{\left[\log \left(1+\frac{x_{i}}{\sigma}\right)\right]^{\beta+1}}{\left[1+\log \left(1+\frac{x_{i}}{\sigma}\right)\right]^{\beta}}
\end{aligned}
$$

The maximum likelihood estimate for the parameters $\alpha, \beta$, and $\sigma$ are given by $\hat{\alpha}, \hat{\beta}$, and $\hat{\sigma}$, are obtained by maximizing the likelihood function in Equation (17). The first-order partial derivatives of (1) with respect to $\alpha, \beta$, and $\sigma$ are

$$
\begin{gathered}
\frac{\partial \ell}{\partial \alpha}=\frac{n}{\alpha}-\sum_{i=1}^{n} \frac{\left[\log \left(1+\frac{x_{i}}{\sigma}\right)\right]^{\beta+1}}{\left[1+\log \left(1+\frac{x_{i}}{\sigma}\right)\right]^{\beta}} \\
\frac{\partial \ell}{\partial \beta}=\sum_{i=1}^{n} \frac{1}{\left(1+\beta+w_{i}\right)}+\sum_{i=1}^{n} \log \left(\frac{w_{i}}{1+w_{i}}\right) \times\left[1-\frac{\alpha w_{i}^{\beta+1}}{\left(1+w_{i}\right)^{\beta}}\right] \\
\frac{\partial \ell}{\partial \sigma}=-\sum_{i=1}^{n} \frac{1}{\left(\sigma+x_{i}\right)}+\sum_{i=1}^{n} \frac{x_{i}}{\sigma\left(\sigma+x_{i}\right)}\left[\frac{\beta+1}{\left(1+w_{i}\right)}-\frac{\beta}{w_{i}}-\frac{1}{\left(1+\beta+w_{i}\right)}\right]+\alpha \sum_{i=1}^{n} \frac{x_{i}}{\sigma\left(\sigma+x_{i}\right)}\left[\frac{\left(1+\beta+w_{i}\right) w_{i}^{\beta}}{\left(1+w_{i}\right)^{\beta+1}}\right],
\end{gathered}
$$

where $w_{i}=\log \left(1+\frac{x_{i}}{\sigma}\right)$.

The MLEs of the three parameters of the $\operatorname{MLM}(\alpha, \beta, \sigma)$ distributions are obtained by setting these above equations to zero and solving them simultaneously. Closed forms of the solutions are not available for the equations (18), (19) and (20). So, iterative methods will be applied to solve these equations numerically.

\subsection{Asymptotic distribution}

Fisher information matrix, a measure of the information content of the data relative to the parameters to be estimated, plays an important role in parameter estimation. The Fisher information matrix $(F)$ can be obtained by taking the expected values of the second-order and mixed partial derivatives of $\ell(\alpha, \beta, \sigma)$ w.r.t. $\alpha, \beta$, and $\sigma$. Since, the analytical expression is hard to compute. Thus, it can be approximated by numerically investing the the $F=\left(F_{i j}\right)$ matrix. The asymptotic $F$ matrix can be given as follows:

$$
F=\left[\begin{array}{ccc}
-\frac{\partial^{2} \ell}{\partial \alpha^{2}} & -\frac{\partial^{2} \ell}{\partial \alpha \partial \beta} & -\frac{\partial^{2} \ell}{\partial \alpha \partial \sigma} \\
-\frac{\partial^{2} \ell}{\partial \alpha \partial \beta} & -\frac{\partial^{2} \ell}{\partial \beta^{2}} & -\frac{\partial^{2} \ell}{\partial \beta \partial \sigma} \\
-\frac{\partial^{2} \ell}{\partial \alpha \partial \sigma} & -\frac{\partial^{2} \ell}{\partial \beta \partial \sigma} & -\frac{\partial^{2} \ell}{\partial \sigma^{2}}
\end{array}\right]
$$


The second and mixed partial derivatives of the log likelihood function are obtained as follows:

$$
\begin{aligned}
& \frac{\partial^{2} \ell}{\partial \alpha^{2}}=-\frac{n}{\alpha^{2}} \\
& \frac{\partial^{2} \ell}{\partial \alpha \partial \beta}=\sum_{i=1}^{n} \log \left(\frac{1+w_{i}}{w_{i}}\right) \times\left[\frac{w_{i}^{\beta+1}}{\left(1+w_{i}\right)^{\beta}}\right] \\
& \frac{\partial^{2} \ell}{\partial \alpha \partial \sigma}=\sum_{i=1}^{n} \frac{x_{i}}{\sigma\left(\sigma+x_{i}\right)} \times\left[\frac{w_{i}^{\beta}\left(1+\beta+w_{i}\right)}{\left(1+w_{i}\right)^{\beta+1}}\right] \\
& \frac{\partial^{2} \ell}{\partial \beta^{2}}=-\sum_{i=1}^{n} \frac{1}{\left(1+\beta+w_{i}\right)^{2}}-\alpha \sum_{i=1}^{n} \log ^{2}\left(\frac{w_{i}}{1+w_{i}}\right)\left[\frac{w_{i}^{\beta+1}}{\left(1+w_{i}\right)^{\beta}}\right] \\
& \frac{\partial^{2} \ell}{\partial \beta \partial \sigma}=\sum_{i=1}^{n} \frac{x_{i}}{\sigma\left(\sigma+x_{i}\right)\left(1+\beta+w_{i}\right)^{2}}-\sum_{i=1}^{n} \frac{x_{i}}{\sigma\left(\sigma+x_{i}\right)} \times\left[\frac{\left(1+w_{i}\right)^{\beta}-\alpha w_{i}^{\beta+1}}{w_{i}\left(1+w_{i}\right)^{\beta+1}}\right] \\
& +\alpha \sum_{i=1}^{n} \frac{x_{i}}{\sigma\left(\sigma+x_{i}\right)}\left[\frac{\left(1+\beta+w_{i}\right) w_{i}^{\beta}}{\left(1+w_{i}\right)^{\beta+1}}\right]\left[\log \left(\frac{w_{i}}{1+w_{i}}\right)\right] \\
& \frac{\partial^{2} \ell}{\partial \sigma^{2}}=\sum_{i=1}^{n} \frac{1}{\left(\sigma+x_{i}\right)^{2}}+\sum_{i=1}^{n} \frac{x_{i}^{2}}{\sigma^{2}\left(\sigma+x_{i}\right)^{2}}\left[\frac{\beta+1}{\left(1+w_{i}\right)^{2}}-\frac{\beta}{w_{i}^{2}}-\frac{1}{\left(1+\beta+w_{i}\right)^{2}}\right] \\
& +\sum_{i=1}^{n} \frac{x_{i}\left(2 \sigma+x_{i}\right)}{\sigma^{2}\left(\sigma+x_{i}\right)^{2}}\left[\frac{\beta}{w_{i}}+\frac{1}{\left(1+\beta+w_{i}\right)}-\frac{\beta+1}{\left(1+w_{i}\right)}\right]-\alpha \sum_{i=1}^{n} \frac{x_{i}^{2}(1+\beta)}{\sigma^{2}\left(x_{i}+\sigma\right)^{2}}\left[\frac{w_{i}^{\beta-1}\left(\beta+w_{i}\right)}{\left(1+w_{i}\right)^{\beta+1}}\right] \\
& -\alpha \sum_{i=1}^{n} \frac{x_{i}}{\sigma^{2}\left(\sigma+x_{i}\right)^{2}}\left[\frac{w_{i}^{\beta}\left(1+\beta+w_{i}\right)\left[\left(2 \sigma+x_{i}\right)\left(1+w_{i}\right)-x_{i}(\beta+1)\right]}{\left(1+w_{i}\right)^{\beta+2}}\right]
\end{aligned}
$$

The variance-covariance matrix is approximated by $M=\left(M_{i j}\right)$ where $M_{i j}=F_{i j}^{-1}$. The asymptotic distribution of MLEs for $\alpha, \beta$, and $\sigma$ can be written as

$$
[(\hat{\alpha}-\alpha),(\hat{\beta}-\beta),(\hat{\sigma}-\sigma)] \sim N_{3}\left(0, F^{-1}(\hat{\theta})\right)
$$

Then the approximate $100(1-k) \%$ confidence intervals for $\alpha, \beta$, and $\sigma$ are given by $\hat{\alpha} \pm z_{\frac{k}{2}} \sqrt{\operatorname{Var}(\hat{\alpha})}$, $\hat{\beta} \pm z_{\frac{k}{2}} \sqrt{\operatorname{Var}(\hat{\beta})}$, and $\hat{\sigma} \pm z_{\frac{k}{2}} \sqrt{\operatorname{Var}(\hat{\sigma})}$, where $\hat{\Theta}=(\hat{\alpha}, \hat{\beta}, \hat{\sigma})$ and $z_{k}$ is the upper $100 \mathrm{k}$-th percentile of the standard normal distribution.

\subsection{Extreme value properties}

Here we study some of the interesting extreme value theoretic properties. The concept of regular variation is an important notion of extreme value theory. Below we show the extreme value results for the MLM distribution that can characterize the asymptotic behavior of extremes along with well grounded statistical theory.

Definition 8. (Maximum domain of attraction) A function $F$ is said to be regularly varying at infinity, if for every $t>0$,

$$
\lim _{x \rightarrow \infty} \frac{1-F(t x)}{1-F(x)}=t^{-\alpha} ; \alpha>0 .
$$


Then we say that $F$ is a function with regularly varying tails with $\alpha>0$ as the tail index and $F$ belongs to the maximum domain of attraction (MDA) of the Frechet distribution with index $\alpha$.

Theorem 3. The CDF (Eqn. 3) of the $M L M$ distribution is a function with regularly varying tails and it belongs to MDA of the Frechet distribution with index $\alpha$.

Proof.

$$
1-F(t x)=\exp \left[-\alpha \cdot \frac{\log ^{\beta+1}\left(1+\frac{t x}{\sigma}\right)}{\left(1+\log \left(1+\frac{t x}{\sigma}\right)\right)^{\beta}}\right] ; t>0
$$

Now, we have (using expansions of $\log (1-x)$ and $\exp (x))$ :

$$
\begin{aligned}
\left(\frac{\log \left(1+\frac{t x}{\sigma}\right)}{1+\log \left(1+\frac{x}{\sigma}\right)}\right)^{\beta} & =\left(1-\frac{1}{1+\log \left(1+\frac{t x}{\sigma}\right)}\right)^{\beta} \\
& =\exp \left[\beta \log \left(1-\frac{1}{1+\log \left(1+\frac{t x}{\sigma}\right)}\right)\right] \\
& =\exp \left[\beta\left(-\frac{1}{\log \left(1+\frac{t x}{\sigma}\right)}+O\left(\frac{1}{\log ^{2}\left(1+\frac{t x}{\sigma}\right)}\right)\right)\right] \\
& =1-\frac{\beta}{\log \left(1+\frac{t x}{\sigma}\right)}+O\left(\frac{\beta}{\log ^{2}\left(1+\frac{t x}{\sigma}\right)}\right)
\end{aligned}
$$

Using Eqn. (27) and (28) together, we get

$$
1-F(t x)=\exp \left[-\alpha \log \left(1+\frac{t x}{\sigma}\right)\left\{1-\frac{\beta}{\log \left(1+\frac{t x}{\sigma}\right)}+O\left(\frac{\beta}{\log ^{2}\left(1+\frac{t x}{\sigma}\right)}\right)\right\}\right]
$$

Similarly for $t=1$, Eqn. (29) becomes

$$
1-F(x)=\exp \left[-\alpha \log \left(1+\frac{x}{\sigma}\right)\left\{1-\frac{\beta}{\log \left(1+\frac{x}{\sigma}\right)}+O\left(\frac{\beta}{\log ^{2}\left(1+\frac{x}{\sigma}\right)}\right)\right\}\right]
$$

Now,

$$
\begin{aligned}
\lim _{x \rightarrow \infty} \frac{1-F(t x)}{1-F(x)} & =\lim _{x \rightarrow \infty} \exp \left[-\alpha \log \left(\frac{1+\frac{t x}{\sigma}}{1+\frac{x}{\sigma}}\right)+O\left(\frac{1}{\log ^{2}\left(1+\frac{t x}{\sigma}\right)}+\frac{1}{\log ^{2}\left(1+\frac{x}{\sigma}\right)}\right)\right] \\
& =\exp (-\alpha \log t) \\
& =t^{-\alpha} .
\end{aligned}
$$

Thus, $F \in M D A\left(\Phi_{\alpha}\right)$.

Now we study the tail-equivalent and heavy-tailed behaviour of the proposed MLM distribution as follows:

Definition 9. (Tail-equivalent) Two distributions $F$ and $G$ are said to be tail-equivalent if

$$
\lim _{x \rightarrow \infty} \frac{1-F(x)}{1-G(x)}=c ; 0<c<\infty
$$

Theorem 4. The $\operatorname{MLM}(\alpha, \beta, \sigma)$ distribution, defined in Eqn. (3), is right tail-equivalent to the power-law distribution. 
Proof. Let $G(x)$ be the CDF of the power-law distribution, i.e.,

$$
1-G(x)=\left(1+\frac{x}{\sigma}\right)^{-\alpha}
$$

and $F(x)$ is the CDF of MLM distribution as given in Eqn.(3). Then,

$$
\begin{aligned}
\lim _{x \rightarrow \infty} \frac{1-F(x)}{1-G(x)} & \left.=\lim _{x \rightarrow \infty} \frac{\exp \left[-\alpha \log \left(1+\frac{x}{\sigma}\right)+\alpha \beta+O\left(\frac{1}{\log \left(1+\frac{x}{\sigma}\right)}\right)\right]}{\exp \left[-\alpha \log \left(1+\frac{x}{\sigma}\right)\right]} \text { (Using Eqn. (330) }\right) \\
& =\lim _{x \rightarrow \infty} \exp \left[\alpha \beta+O\left(\frac{1}{\log \left(1+\frac{x}{\sigma}\right)}\right)\right] \\
& =\exp (\alpha \beta) \\
& =c<\infty
\end{aligned}
$$

Definition 10. (Heavy-tailed distribution) A distribution function $F$ is heavy-tailed if

$$
\lim _{x \rightarrow \infty} \exp \{\lambda x\}(1-F(x))=\infty \text {, for any } \lambda>0 .
$$

Theorem 5. The $M L M(\alpha, \beta, \sigma)$ distributions, defined in Eqn. (3), are heavy-tailed distributions.

Proof.

$$
\begin{aligned}
\lim _{x \rightarrow \infty} \exp \{\lambda x\}(1-F(x)) & =\lim _{x \rightarrow \infty} \exp \left[\lambda x-\alpha \log \left(1+\frac{x}{\sigma}\right)+\alpha \beta+O\left(\frac{1}{\log \left(1+\frac{x}{\sigma}\right)}\right)\right] \\
& =\infty
\end{aligned}
$$

since $\log \left(1+\frac{x}{\sigma}\right) \asymp x^{\epsilon}$ for any $\epsilon>0$ and for sufficiently large $x$.

There are two other important class of distributions [44] viz. the class $\mathbb{D}$ of dominated-variation distributions and and the class $\mathbb{L}$ of long-tailed distributions that are used in the risk theory and queueing theory. The proposed MLM distributions also follows these two properties.

Definition 11. A distribution $F$ belong to the class $\mathbb{D}$ of dominated-variation distributions if

$$
\limsup _{x \rightarrow \infty} \frac{1-F(x)}{1-F(2 x)}<\infty .
$$

Theorem 6. If $\alpha>0$, then $\operatorname{MLM}(\alpha, \beta, \sigma)$ distribution, defined in Eqn. (3), belongs to the class $\mathbb{D}$ of dominated-variation distributions. 
Proof.

$$
\begin{aligned}
\lim _{x \rightarrow \infty} \frac{1-F(x)}{1-F(2 x)} & =\lim _{x \rightarrow \infty} \frac{\exp \left[-\alpha \log \left(1+\frac{x}{\sigma}\right)+\alpha \beta+O\left(\frac{1}{\log \left(1+\frac{x}{\sigma}\right)}\right)\right]}{\exp \left[-\alpha \log \left(1+\frac{2 x}{\sigma}\right)+\alpha \beta+O\left(\frac{1}{\log \left(1+\frac{2 x}{\sigma}\right)}\right)\right]} \\
& =\lim _{x \rightarrow \infty} \exp \left[\alpha \log \left(\frac{1+\frac{2 x}{\sigma}}{1+\frac{x}{\sigma}}\right)+O\left(\frac{1}{\log \left(1+\frac{x}{\sigma}\right)}+\frac{1}{\log \left(1+\frac{2 x}{\sigma}\right)}\right)\right] \\
& =\exp (\alpha \log 2) \\
& =2^{\alpha}<\infty .
\end{aligned}
$$

where $\alpha>0$.

Definition 12. A distribution $F$ is said to belong to the class $\mathbb{L}$ of long-tailed distributions if $F$ has right unbounded support and, for any fixed $y>0$,

$$
\lim _{x \rightarrow \infty} \frac{1-F(x+y)}{1-F(x)}=1 .
$$

Theorem 7. The $\operatorname{MLM}(\alpha, \beta, \sigma)$ distribution, defined in Eqn. (3), belongs to the class $\mathbb{L}$ of long-tailed distributions.

Proof.

$$
\begin{aligned}
\lim _{x \rightarrow \infty} \frac{1-F(x+y)}{1-F(x)} & =\lim _{x \rightarrow \infty} \exp \left[-\alpha \log \left(1+\frac{(y / \sigma)}{\left(1+\frac{x}{\sigma}\right)}\right)+O\left(\frac{1}{\log \left(1+\frac{x}{\sigma}\right)}+\frac{1}{\log \left(1+\frac{x+y}{\sigma}\right)}\right)\right] \\
& =1, \quad \text { since } \sigma>0 .
\end{aligned}
$$

We have shown that the proposed MLM distributions are heavy-tailed and also possess the additional regularity property of subexponentiality [45] as given below. Essentially this corresponds to good tail behaviour under the operation of convolution.

Definition 13. (Subexponential distribution) We say that a distribution $F$ is subexponential if

$$
\lim _{x \rightarrow \infty} \frac{1-F * F(x)}{1-F(x)}=2,
$$

where $*$ denotes the convolution operation.

Theorem 8. The $M L M(\alpha, \beta, \sigma)$ distribution, defined in Eqn. (3), is subexponential.

Proof. Form Theorem [6] and Theorem 7, the $\operatorname{MLM}(\alpha, \beta, \sigma)$ distribution belongs to $\mathbb{D} \cap \mathbb{L}$. Using [46], $\mathbb{D} \cap \mathbb{L} \subset \mathbb{S}$, where $S$ is the class of subsexponetial distribution. Hence the theorem.

Definition 14. (Von-Mises type function) A distribution function $F$ is called a Von-Mises type function if

$$
\lim _{x \uparrow r(F)} x \frac{d}{d x}\left[\frac{1-F(x)}{x f(x)}\right]=0,
$$

where $r(F)=\sup \{x: F(x)<1\}$ denotes the right extremity of the distribution function $F$ [44]. 
Theorem 9. The $\operatorname{MLM}(\alpha, \beta, \sigma)$ distribution, defined in Eqn. (3), satisfies the Von-Mises condition.

Proof.

$$
\begin{aligned}
\lim _{x \rightarrow \infty} x \frac{d}{d x}\left[\frac{1-F(x)}{x f(x)}\right] & =\frac{\alpha}{\sigma} \lim _{x \rightarrow \infty} x \frac{d}{d x}\left[\frac{\left(1+\frac{x}{\sigma}\right)\left[1+\log \left(1+\frac{x}{\sigma}\right)\right]^{\beta+1}}{x\left[\beta+1+\log \left(1+\frac{x}{\sigma}\right)\right]\left[\log \left(1+\frac{x}{\sigma}\right)\right]^{\beta}}\right] \\
& =\frac{\alpha}{\sigma} \lim _{x \rightarrow \infty} \frac{\left[1+\log \left(1+\frac{x}{\sigma}\right)\right]^{\beta+1}}{\left[\beta+1+\log \left(1+\frac{x}{\sigma}\right)\right]\left[\log \left(1+\frac{x}{\sigma}\right)\right]^{\beta}} \\
& \times\left[-\frac{1}{x}+\frac{1}{\sigma\left(1+\log \left(1+\frac{x}{\sigma}\right)\right)}-\frac{1}{\sigma\left(1+\beta+\log \left(1+\frac{x}{\sigma}\right)\right)}-\frac{\beta}{\sigma \log \left(1+\frac{x}{\sigma}\right)}\right] \\
& =0 .
\end{aligned}
$$

\section{Experimental Analysis}

\subsection{Description of data sets}

We present here the results of fitting modified Lomax (MLM) distribution over 50 real-worlld complex networks [4, 48] coming from broad variety of different disciplines such as Social Networks, Collaboration Networks, Communication Networks, Citation Networks, Temporal Networks, Web Graphs, Product copurchasing Networks, Biological Networks, Brain Networks, etc. Please go through the supplementary materials for more details about the data sets under consideration. Some statistical measures of the data sets and the detailed experimentation of the performances of the proposed MLM distribution compared to the other common power-law related distribution such as Lomax, Pareto, Log-normal, power-law cutoff, Exponential and Poisson are discussed in the following sub sections.

\subsection{Performance measures}

Here we use some evaluation measures which justify that the degree distribution of a real-world complex network can plausibly been drawn from the proposed MLM distribution. As here the actual distribution is discrete, we can quantify the goodness-of-fit test (i.e., how closely a hypothesized distribution resembles the actual distribution) by calculating the Chi-square statistic value based on bootstrap resampling by generating 50000 synthetic data sets. The Chi-square test will return a $p$ value which quantifies the probability that our data were drawn from the hypothesized distribution. If the $p$ value is small (less than the significance level), we can reject the null hypothesis that the data come from the MLM distribution. We have also computed few other statistical measures such as KL-divergence, RMSE and MAE for quantifying the goodness-of-fit of the proposed MLM distribution model in comparison to the other standard distribution functions related to other heavy-tailed distributions.

\subsection{Analysis of results}

Table 1 represents some of the statistical measures corresponding to the network data and also provides the statistical evidences of the proposed fitting over the node degree distribution in the whole range using MLM distribution. CV is also calculated corresponding to each of the degree distribution data and it gives us the sufficient condition for the existence of the global maximum at finite point of the $M L M(\alpha, \beta, \sigma)$ distribution. From Table 1 it is clear that that the value of $\mathrm{CV}$ is greater than 1 in all the network data sets under consideration. Thus it confirms that the maximum likelihood estimates for the parameters $(\alpha, \beta, \sigma)$ of the proposed MLM distribution attain at the finite points which has been theoretically described in Section 441.1. To estimate the parameters $(\alpha, \beta, \sigma)$ of the MLM distribution numerically, we have used "optim" function along with the quasi-Newton L-BFGS-B algorithm in R statistical software by taking the initial parameters value $(\alpha, \beta, \sigma)=(1,0,1)$. The estimated values of the parameters for all the data sets satisfied 
Table 1: Performance of the proposed MLM model over different real-worlld networks

\begin{tabular}{|c|c|c|c|c|c|c|c|c|c|c|}
\hline \multicolumn{2}{|r|}{$\begin{array}{l}\text { Data } \\
\text { sets }\end{array}$} & \multirow[t]{2}{*}{$\begin{array}{l}\text { No. of } \\
\text { nodes }\end{array}$} & \multirow[t]{2}{*}{$\begin{array}{l}\text { No. of } \\
\text { edges }\end{array}$} & \multicolumn{3}{|c|}{ Stat. Prop. } & \multicolumn{3}{|c|}{$\begin{array}{l}\text { Estimated } \\
\text { parameters }\end{array}$} & \multirow{2}{*}{$\begin{array}{c}\text { Bootstrap } \\
\text { chi-square } \\
\text { value } \\
(p)\end{array}$} \\
\hline & & & & $s$ & $\mu$ & $\frac{s}{\mu}$ & $\widehat{\alpha}$ & $\widehat{\beta}$ & $\widehat{\sigma}$ & \\
\hline \multirow{11}{*}{$\begin{array}{c}\text { Social } \\
\text { Networks }\end{array}$} & ego-Twitter(In) & 81,306 & $1,768,149$ & 57.965 & 21.747 & 2.6654 & 1.9922 & -0.3591 & 30.543 & 0.9920 \\
\hline & ego-Gplus(In) & 107,614 & $13,673,453$ & 1404.8 & 283.42 & 4.9568 & 0.7108 & -0.4983 & 23.077 & 0.9963 \\
\hline & soc-Slashdot & 70,068 & 358,647 & 35.069 & 10.237 & 3.426 & 0.8663 & -0.6228 & 1.0461 & 0.9955 \\
\hline & soc-Delicious(In) & 536,108 & $1,365,961$ & 39.826 & 10.673 & 3.7312 & 1.3630 & -0.6819 & 5.3709 & 0.9960 \\
\hline & soc-Digg(In) & 770,799 & $5,907,132$ & 166.61 & 46.584 & 3.5765 & 0.7931 & -0.6928 & 5.5163 & 0.9890 \\
\hline & soc-Academia & 200,169 & $1,398,063$ & 48.297 & 14.259 & 3.3871 & 2.7429 & -0.3737 & 36.644 & 0.6087 \\
\hline & LiveJournal(In) & $4,847,571$ & $68,993,773$ & 44.969 & 15.368 & 2.926 & 2.6892 & -0.7272 & 51.933 & 0.8983 \\
\hline & Dogster-Friendship & 426,821 & $8,546,581$ & 284.06 & 40.033 & 7.095 & 1.5634 & 0.3108 & 14.057 & 0.9500 \\
\hline & Higgs-Twitter(In) & 456,626 & $14,855,842$ & 350.91 & 54.786 & 6.4051 & 1.6797 & -0.0347 & 36.204 & 0.9870 \\
\hline & Artist-Facebook & 50,615 & 819,307 & 63.427 & 32.366 & 1.9596 & 2.0117 & -0.1445 & 39.337 & 0.9812 \\
\hline & Athletes-Facebook & 13,866 & 86,859 & 17.978 & 12.438 & 1.4453 & 3.1229 & 0.1406 & 21.180 & 0.9640 \\
\hline \multirow{4}{*}{$\begin{array}{l}\text { Citation } \\
\text { Networks }\end{array}$} & cit-HepTh(In) & 27,770 & 352,807 & 43.139 & 15.220 & 2.8342 & 1.8410 & -0.3093 & 16.416 & 0.8730 \\
\hline & cit-HepPh(In) & 34,546 & 421,578 & 27.286 & 14.933 & 1.8271 & 2.5553 & -0.3622 & 34.349 & 0.9900 \\
\hline & cit-Patents(In) & $3,774,768$ & $16,518,948$ & 6.9125 & 5.0687 & 1.3637 & 4.4822 & -0.2534 & 21.689 & 0.8080 \\
\hline & cit-Citeseer(In) & 227,320 & 814,134 & 9.8260 & 5.4322 & 1.8088 & 2.2630 & -0.2788 & 7.4150 & 0.6350 \\
\hline \multirow{5}{*}{$\begin{array}{l}\text { Collaboration } \\
\text { Networks }\end{array}$} & ca-CondMat & 23,133 & 93,497 & 10.671 & 8.0189 & 1.3308 & 3.1068 & 0.3615 & 10.5353 & 0.9896 \\
\hline & ca-AstroPh & 18,772 & 198,110 & 30.568 & 21.103 & 1.4484 & 16.434 & 37.276 & 0.0101 & 0.9990 \\
\hline & ca-GrQc & 5,242 & 14,496 & 7.9186 & 5.5284 & 1.4322 & 2.2624 & 3.5861 & 0.6765 & 0.7849 \\
\hline & $\mathrm{ca}-\mathrm{HepPh}$ & 12,008 & 118,521 & 46.654 & 19.696 & 2.3687 & 0.9798 & 2.8780 & 0.6791 & 0.8163 \\
\hline & ca-HepTh & 9,877 & 25,998 & 6.1867 & 5.2618 & 1.1757 & 2.9417 & 5.2791 & 0.4825 & 0.9332 \\
\hline \multirow{5}{*}{$\begin{array}{l}\text { Web } \\
\text { Graphs }\end{array}$} & Google(In) & 875,713 & $5,105,039$ & 43.320 & 7.1444 & 6.0634 & 1.1999 & -0.6399 & 2.0429 & 0.9780 \\
\hline & BerkStan(In) & 685,230 & $7,600,595$ & 300.08 & 12.316 & 24.364 & 1.4129 & 1.8449 & 0.7592 & 0.6250 \\
\hline & Wikipedia2009(In) & $1,864,433$ & $4,507,315$ & 12.846 & 4.8903 & 2.6268 & 1.3988 & -0.6291 & 1.9658 & 0.9891 \\
\hline & WikipediaLinkFr(In) & $4,906,478$ & $113,122,279$ & 1864.4 & 48.608 & 38.356 & 1.0988 & -0.7123 & 9.8888 & 0.9152 \\
\hline & Hudong(In) & $1,984,484$ & $14,869,483$ & 199.28 & 16.467 & 12.101 & 1.1567 & 10.921 & 0.0013 & 0.9883 \\
\hline \multirow{5}{*}{$\begin{array}{l}\text { Biological } \\
\text { Networks }\end{array}$} & Yeast-PPIN & 2,361 & 7,182 & 8.0800 & 6.0838 & 1.3281 & 10.535 & -0.4527 & 175.29 & 0.9930 \\
\hline & Diseasome & 3,926 & 7,823 & 9.1009 & 5.5334 & 1.6447 & 10.9688 & -0.9493 & 134.52 & 0.8090 \\
\hline & Bio-Mouse-Gene & 45,101 & $14,506,199$ & 856.67 & 643.27 & 1.3317 & $6.3 \mathrm{e}-08$ & $-1.2 \mathrm{e}-02$ & $2.1 \mathrm{e}+00$ & 0.9898 \\
\hline & Bio-Dmela & 7,393 & 25569 & 10.782 & 6.9170 & 1.5587 & 14.979 & -0.5053 & 498.27 & 0.9806 \\
\hline & Bio-WormNet-v3 & 16,347 & 762,822 & 138.17 & 93.328 & 1.4805 & 5.6496 & -0.9801 & 704.71 & 0.9938 \\
\hline \multirow{3}{*}{$\begin{array}{c}\text { Product } \\
\text { co-purchasing } \\
\text { networks }\end{array}$} & amazon0601(In) & 403,394 & $3,387,388$ & 15.279 & 8.3989 & 1.8191 & 3.8261 & -.7137 & 19.522 & 0.6010 \\
\hline & amazon0505(In) & 410,236 & $3,356,828$ & 15.313 & 8.1826 & 1.8714 & 3.8367 & -0.8006 & 19.984 & 0.6880 \\
\hline & amazon0312(In) & 400,727 & $3,200,444$ & 15.073 & 7.9865 & 1.8873 & 3.7631 & -0.8179 & 18.747 & 0.5890 \\
\hline \multirow{4}{*}{$\begin{array}{l}\text { Temporal } \\
\text { Networks }\end{array}$} & sx-mathoverflow(In) & 24,818 & 506,550 & 31.476 & 10.424 & 3.0195 & 1.4452 & 2.4236 & 0.8241 & 0.9846 \\
\hline & sx-stackoverflow(In) & $2,601,977$ & $63,497,050$ & 186.00 & 27.647 & 6.7278 & 1.0218 & -0.8224 & 4.4865 & 0.9490 \\
\hline & sx-superuser(In) & 194,085 & $1,443,339$ & 23.782 & 5.8239 & 4.0836 & 1.7401 & 2.1405 & 0.7284 & 0.9780 \\
\hline & sx-askubuntu (In) & 159,316 & 964,437 & 18.404 & 4.3856 & 4.1966 & 2.1923 & 2.2069 & 0.7665 & 0.9300 \\
\hline \multirow{3}{*}{$\begin{array}{l}\text { Communication } \\
\text { Networks }\end{array}$} & Email-Enron & 36,692 & 183,831 & 36.100 & 10.021 & 3.6027 & 1.2417 & -0.1275 & 2.9045 & 0.9641 \\
\hline & Wiki-Talk(In) & $2,394,385$ & $5,021,410$ & 12.259 & 2.1195 & 5.7844 & 1.5167 & -0.2846 & 0.0016 & 0.9900 \\
\hline & Rec-Libimseti(In) & 220,970 & $17,359,346$ & 413.71 & 102.85 & 4.0227 & 2.5008 & -0.8496 & 331.18 & 0.9670 \\
\hline \multirow{5}{*}{$\begin{array}{l}\text { Ground-truth } \\
\text { Networks }\end{array}$} & Wiki-Topcats & $1,791,489$ & $28,511,807$ & 283.78 & 15.915 & 17.831 & 1.1811 & 0.1998 & 2.6412 & 0.8310 \\
\hline & com-Friendster & $65,608,366$ & $1,806,067,135$ & 137.81 & 55.056 & 2.5031 & 4.5863 & -0.9188 & 590.01 & 0.9000 \\
\hline & com-LiveJournal & $\begin{array}{l}3,997,962 \\
3\end{array}$ & $34,681,189$ & 42.957 & 17.349 & 2.4759 & 2.8206 & $\begin{array}{l}-0.9100 \\
-0.6020\end{array}-100$ & 65.638 & 0.7980 \\
\hline & com-Orkut & $3,072,441$ & $117,185,083$ & 154.78 & 76.281 & 2.0291 & 3.7049 & 0.1292 & 167.93 & 0.9890 \\
\hline & com-Youtube & $1,134,890$ & $2,987,624$ & 50.754 & 5.2650 & 9.6398 & 1.6113 & 8.3355 & 0.0094 & 0.8410 \\
\hline \multirow{5}{*}{$\begin{array}{c}\text { Brain } \\
\text { Networks }\end{array}$} & Human25890-session1 & 177,584 & $15,669,036$ & 319.01 & 176.47 & 1.8078 & 1.6098 & -0.2076 & 168.75 & 0.8710 \\
\hline & Human $25890-$ session 2 & 723,881 & $158,147,409$ & 667.91 & 436.94 & 1.5286 & 14.423 & -0.3466 & 18886.3 & 0.9980 \\
\hline & Human25864-session2 & 692,957 & $133,727,516$ & 554.48 & 385.96 & 1.4366 & 16.250 & -0.3379 & 19217.8 & 0.9660 \\
\hline & Human25913-session2 & 726,197 & $183,978,766$ & 446.92 & 258.99 & 1.7256 & 7.1013 & -0.4681 & 5779.8 & 0.9290 \\
\hline & Human25886-session1 & 780,185 & $158,184,747$ & 558.41 & 405.50 & 1.3771 & 21.591 & -0.3119 & 26975.9 & 0.9768 \\
\hline
\end{tabular}

the condition, i.e., $(\alpha>0, \beta>-1$ and $\sigma>0)$ as clearly seen in Table 1 for the complete characterization of the proposed MLM distribution. Empirically it is observed that in almost all the cases the estimated value of the parameter $\sigma$ attains the higher values as compared to the estimated value of $\alpha$. On the other hand, the estimated value of the parameter $\beta$ lies between $(0,1)$ lies between -1 and 1 except a few which can be clearly seen from Table 1 .

Furthermore, we leverage one of the popular statistical method viz. bootstrapping chi-square test to evaluate the goodness-of-fit test of the proposed MLM distribution. From Table 1 it is clear that the proposed MLM distribution produces higher p values (i.e. closure to 1) in almost all the data sets which suggest that the null hypothesis i.e. the data drawn from MLM distribution cannot be ruled out at the 0.05 level of significance. This indicates that the observed degree distribution is plausibly drawn from the MLM distribution. Thus from Table 1 it can be concluded that the proposed MLM distribution is effective in modeling the entire degree distribution of real-worlld complex networks without ignoring some of the lower degree nodes as oppose to the procedure of fitting power law distribution. In addition, we also used some other statical measures viz. KLD, RMSE and MAE in order to compare the performance of the proposed MLM distribution with the each of the other common power-law related distributions as given in the following Tables 2 and 3 .

Tables 2 and 3 depict the values of different statistical measures (viz. RMSE, MAE and KLD) which has been used for the measure of performances of the MLM distribution in comparison to the competitive distributions while modeling the data. RMSE and MAE are two different variants, carrying information about the differences between actual and predicted degree frequencies corresponding to a network. Higher similarity between actual and mapped distributions is achieved by generating smaller values of RMSE and 
Table 2: Table of different statistical measures of different competitive models over real-worlld networks

\begin{tabular}{|c|c|c|c|c|c|c|c|c|c|c|c|c|c|}
\hline \multicolumn{2}{|r|}{$\begin{array}{l}\text { Data } \\
\text { sets }\end{array}$} & \multicolumn{3}{|c|}{ MLM } & \multicolumn{3}{|c|}{ Lomax } & \multicolumn{3}{|c|}{ Power-law } & \multicolumn{3}{|c|}{ Pareto } \\
\hline & & RMSE & KLD & MAE & RMSE & KLD & MAE & RMSE & KLD & MAE & RMSE & KLD & MAE \\
\hline \multirow{11}{*}{$\begin{array}{c}\text { Social } \\
\text { Networks }\end{array}$} & ego-Twitter(In) & 16.800 & 0.00819 & 1.3498 & 29.366 & 0.01354 & 2.4701 & 204.35 & 0.1831 & 10.847 & 354.25 & 0.2857 & 15.603 \\
\hline & ego-Gplus(In) & 1.6115 & 0.05601 & 0.1825 & 10.491 & 0.06444 & 0.3033 & 53.064 & 0.2299 & 0.9221 & 86.955 & 0.3113 & 1.1847 \\
\hline & soc-Slashdot & 31.527 & 0.01365 & 2.3951 & 32.065 & 0.014102 & 2.4658 & 247.87 & 0.1007 & 10.074 & 247.84 & 0.1007 & 10.073 \\
\hline & soc-Delicious(In) & 79.809 & 0.00839 & 3.7730 & 91.993 & 0.01326 & 4.8060 & 349.66 & 0.2021 & 14.867 & 471.02 & 0.1349 & 17.874 \\
\hline & soc-Digg(In) & 13.634 & 0.02182 & 0.8440 & 24.841 & 0.02391 & 1.0269 & 208.01 & 0.1601 & 4.2185 & 212.87 & 0.1601 & 4.2312 \\
\hline & soc-Academía & 16.323 & 0.00351 & 0.5705 & 48.951 & 0.01019 & 1.6178 & 229.54 & 0.2027 & 6.3889 & 440.15 & 0.274 & 10.464 \\
\hline & LiveJournal(In) & 243.99 & $6.13 \mathrm{e}-04$ & 5.4026 & 1764.9 & 0.02111 & 54.400 & 5025.2 & 0.1614 & 127.98 & 8100.9 & 0.1785 & 164.18 \\
\hline & Dogster-Friendship & 32.203 & 0.01328 & 0.8502 & 36.449 & 0.01700 & 1.0755 & 358.27 & 0.2926 & 5.6815 & 549.57 & 0.4618 & 7.6734 \\
\hline & Higgs-Twitter(In) & 19.821 & 0.00785 & 0.4710 & 20.609 & 0.00793 & 0.4621 & 260.32 & 0.2492 & 4.8938 & 524.96 & 0.4806 & 7.6938 \\
\hline & Artist-Facebook & 11.708 & 0.01079 & 2.1381 & 12.923 & 0.01199 & 2.6173 & 100.49 & 0.1643 & 14.552 & 350.05 & 0.4010 & 26.467 \\
\hline & Athletes-Facebook & 4.4304 & 0.00879 & 1.3252 & 9.2379 & 0.00966 & 1.8260 & 100.16 & 0.2049 & 13.387 & 204.91 & 0.4164 & 23.839 \\
\hline \multirow{4}{*}{$\begin{array}{l}\text { Citation } \\
\text { Networks }\end{array}$} & cit-HepTh(In) & 3.2640 & 0.01354 & 0.5071 & 7.9393 & 0.01585 & 0.7585 & 73.531 & 0.1741 & 4.0821 & 122.79 & 0.2566 & 5.997 \\
\hline & cit-HepPh(In) & 9.6810 & 0.00821 & 1.9016 & 21.303 & 0.01317 & 3.1135 & 128.55 & 0.1825 & 13.234 & 257.41 & 0.2689 & 21.445 \\
\hline & cit-Patents(In) & 445.80 & $1.61 \mathrm{e}-04$ & 47.603 & 2577.5 & 0.00192 & 230.35 & $27.5 \mathrm{~K}$ & 0.2266 & 2049.5 & $34.8 \mathrm{~K}$ & 0.2366 & 2533.2 \\
\hline & cit-Citeseer(In) & 40.728 & 0.00228 & 3.3778 & 28.032 & 0.00278 & 3.3902 & 889.88 & 0.3308 & 49.467 & 1156.2 & 0.2916 & 62.026 \\
\hline \multirow{5}{*}{$\begin{array}{c}\text { Collaboration } \\
\text { Networks }\end{array}$} & ca-CondMat & 14.830 & 0.00479 & 4.1570 & 36.094 & 0.00814 & 7.3904 & 107.86 & 0.1025 & 26.092 & 469.12 & 0.3738 & 63.075 \\
\hline & ca-AstroPh & 23.890 & 0.02756 & 5.9796 & 32.799 & 0.03457 & 7.4448 & 92.255 & 0.1753 & 15.158 & 251.03 & 0.3816 & 27.707 \\
\hline & ca-GrQc & 15.850 & 0.03055 & 7.2247 & 35.935 & 0.04013 & 12.286 & 124.24 & 0.2554 & 27.137 & 202.33 & 0.2741 & 44.221 \\
\hline & ca-HepPh & 13.944 & 0.06959 & 4.1919 & 19.607 & 0.07266 & 4.7763 & 75.071 & 0.1769 & 8.0906 & 144.39 & 0.2569 & 14.668 \\
\hline & ca-HepTh & 23.280 & 0.00896 & 10.851 & 61.797 & 0.01353 & 20.391 & 268.91 & 0.2346 & 66.108 & 437.19 & 0.2829 & 106.36 \\
\hline \multirow{5}{*}{$\begin{array}{c}\text { Web } \\
\text { Graphs }\end{array}$} & Google(In) & 360.62 & 0.01368 & 13.845 & 337.68 & 0.01546 & 14.201 & 1809.1 & 0.124 & 45.023 & 1809.2 & 0.124 & 45.023 \\
\hline & BerkStan(In) & 71.819 & 0.03116 & 0.9478 & 105.20 & 0.0346 & 1.1962 & 615.03 & 0.1863 & 4.0722 & 615.01 & 0.1863 & 4.0721 \\
\hline & Wikipedia2009(In) & 86.510 & 0.00169 & 7.7498 & 103.94 & 0.00197 & 8.5289 & 4371.9 & 0.1352 & 164.58 & 4371.9 & 0.1352 & 164.58 \\
\hline & WikipediaLinkFr(In) & 124.98 & 0.01776 & 0.3174 & 146.14 & 0.03082 & 0.4465 & 248.09 & 0.1518 & 0.7857 & 397.39 & 0.1521 & 1.0815 \\
\hline & Hudong(In) & 8.0517 & 0.00433 & 0.2508 & 25.163 & 0.00525 & 0.4600 & 587.21 & 0.0868 & 4.6828 & 587.22 & 0.0868 & 4.6828 \\
\hline \multirow{5}{*}{$\begin{array}{l}\text { Biological } \\
\text { Networks }\end{array}$} & Yeast-PPIN & 4.3766 & 0.01487 & 2.6321 & 12.651 & 0.02389 & 5.4529 & 75.325 & 0.1999 & 19.013 & 77.455 & 0.1998 & 19.079 \\
\hline & Diseasome & 8.8683 & 0.08000 & 2.7445 & 12.451 & 0.10202 & 3.4575 & 26.006 & 0.2248 & 5.3567 & 26.005 & 0.2248 & 5.3566 \\
\hline & Bio-Mouse-Gene & 7.6919 & 0.18941 & 2.2557 & 14.654 & 0.19473 & 2.3919 & 41.371 & 0.4566 & 3.9018 & 92.539 & 0.5373 & 4.7342 \\
\hline & Bio-Dmela & 16.173 & 0.01305 & 4.0968 & 10.579 & 0.01759 & 3.8219 & 143.71 & 0.1907 & 21.415 & 143.67 & 0.1907 & 21.414 \\
\hline & Bio-WormNet-v3 & 14.054 & 0.04648 & 2.6066 & 13.018 & 0.09249 & 3.7468 & 46.259 & 0.2761 & 6.8867 & 101.89 & 0.3744 & 9.0163 \\
\hline \multirow{3}{*}{$\begin{array}{c}\text { Product } \\
\text { co-purchasing } \\
\text { networks } \\
\end{array}$} & amazon0601(In) & 94.347 & 0.00374 & 8.1863 & 147.602 & 0.00695 & 10.928 & 1495.4 & 0.2708 & 70.281 & 2539.8 & 0.4022 & 114.59 \\
\hline & amazon0505(In) & 109.95 & 0.00412 & 9.1836 & 94.048 & 0.00499 & 8.7882 & 1572.9 & 0.2463 & 73.003 & 2494.5 & 0.3711 & 111.56 \\
\hline & amazon0312(In) & 100.89 & 0.00430 & 8.5465 & 92.525 & 0.00495 & 8.5742 & 1564.4 & 0.2425 & 71.875 & 2462.9 & 0.3686 & 109.21 \\
\hline \multirow{4}{*}{$\begin{array}{l}\text { Temporal } \\
\text { Networks }\end{array}$} & sx-mathoverflow(In) & 19.706 & 0.01879 & 2.4647 & 38.764 & 0.02621 & 3.7877 & 213.91 & 0.2131 & 13.600 & 213.82 & 0.2132 & 13.612 \\
\hline & sx-stackoverflow(In) & 39.654 & 0.00336 & 0.8694 & 62.254 & 0.00345 & 1.0741 & 1877.5 & 0.2016 & 14.007 & 1884.7 & 0.2017 & 14.017 \\
\hline & sx-superuser(In) & 79.777 & 0.00654 & 4.5409 & 136.85 & 0.01045 & 6.8313 & 900.04 & 0.1808 & 33.837 & 900.33 & 0.1808 & 33.839 \\
\hline & sx-askubuntu(In) & 106.04 & 0.01100 & 6.2022 & 176.58 & 0.01707 & 9.3509 & 949.66 & 0.2091 & 39.419 & 949.73 & 0.2091 & 39.420 \\
\hline \multirow{3}{*}{$\begin{array}{c}\text { Communication } \\
\text { Networks }\end{array}$} & Email-Enron & 74.667 & 0.03523 & 5.2075 & 76.155 & 0.03531 & 5.2347 & 246.51 & 0.1779 & 14.886 & 245.25 & 0.1778 & 14.859 \\
\hline & Wiki-Talk(In) & 670.47 & 0.00356 & 25.871 & 671.76 & 0.00357 & 25.898 & 9669.4 & 0.3376 & 293.63 & 9669.4 & 0.3376 & 293.63 \\
\hline & Rec-Libimseti(In) & 23.341 & 0.02163 & 0.4953 & 66.434 & 0.09978 & 1.7923 & 77.081 & 0.2198 & 2.1486 & 133.91 & 0.2096 & 2.7441 \\
\hline \multirow{5}{*}{$\begin{array}{l}\text { Ground-truth } \\
\text { Networks }\end{array}$} & Wiki-Topcats & 11.375 & 0.00190 & 0.1011 & 14.955 & 0.00201 & 0.1347 & 565.21 & 0.1377 & 2.6145 & 930.44 & 0.1612 & 3.8073 \\
\hline & com-Friendster & 8266.8 & 0.00126 & 411.15 & $41.69 \mathrm{~K}$ & 0.06401 & 3385.2 & $71.5 \mathrm{~K}$ & 0.1498 & 4575.6 & $129 \mathrm{~K}$ & 0.1498 & 5591.7 \\
\hline & com-LiveJournal & 165.79 & 0.00084 & 6.9832 & 1741.3 & 0.02462 & 50.318 & 4102.9 & 0.1823 & 106.85 & 7116.4 & 0.2147 & 150.46 \\
\hline & com-Orkut & 197.89 & 0.00793 & 7.0113 & 207.43 & 0.01049 & 9.9761 & 2443.6 & 0.5498 & 80.712 & 4299.3 & 0.8033 & 101.64 \\
\hline & com-Youtube & 53.288 & 0.00122 & 0.6984 & 81.409 & 0.00175 & 1.0862 & 1380.5 & 0.1342 & 15.690 & 1380.5 & 0.1342 & 15.691 \\
\hline \multirow{5}{*}{$\begin{array}{c}\text { Brain } \\
\text { Networks }\end{array}$} & Human25890-session1 & 17.309 & 0.01920 & 3.7439 & 19.545 & & 4.0023 & 305.41 & 0.3397 & 22.537 & 588.71 & 0.5598 & 30.333 \\
\hline & Human25890-session2 & 46.276 & 0.01024 & 5.8781 & 97.122 & 0.04774 & 15.303 & 794.95 & 0.4462 & 50.379 & 1623.8 & 0.6513 & 63.754 \\
\hline & Human25864-session2 & 64.037 & 0.01321 & 9.8670 & 111.45 & 0.05335 & 20.876 & 1120.3 & 0.4967 & 68.172 & 1711.3 & 0.6419 & 78.736 \\
\hline & Human25913-session2 & 112.661 & 0.01347 & 11.574 & 119.54 & 0.04719 & 20.971 & 904.76 & 0.2764 & 58.999 & 1892.4 & 0.4566 & 81.206 \\
\hline & Human25886-session 1 & 65.181 & 0.01471 & 12.051 & 116.78 & 0.05476 & 23.396 & 978.66 & 0.4664 & 69.517 & 1873.6 & 0.6805 & 86.597 \\
\hline
\end{tabular}

MAE. From Tables 2]and3, it is clear that the proposed MLM distribution provides smaller RMSE and MAE values compared to other competitive distributions in almost all the networks except a few where the powerlaw cutoff distribution outperforms the others. The worst performance observed for the poisson distribution in minimizing the RMSE and MAE values compared to the other competing distributions over all the realworlld networks as clearly seen from Table 3. The Kullback-Leibler divergence (KLD), or relative entropy, is a quantity which measures the dissimilarity between two probability distributions. Thus the smaller value of KLD represents the higher similarity between the actual and the predicted distribution. From Tables 2 and 3 it is clear that the proposed MLM distribution generates smaller KLD values compared to other competitive distributions in almost all the networks except a few where power-law cutoff distribution outperforms the others. This indicates that the observed degree distribution satisfactorily matches the proposed MLM distribution in almost all the networks. Note that, in terms of KLD, the Poisson and Exponential distributions always perform worse than the others in all the networks as in the case RMSE and MAE. The performance of the proposed MLM distribution is always superior to the competitive in terms of KLD over almost all the networks. Thus overall, by considering RMSE, MAE and KLD values, the performance of the proposed MLM distribution for all the networks is found to be better than the other competing distributions which suggest that the observed distribution plausibly comes from the proposed MLM distribution. 
Table 3: Table of different statistical measures of different competitive models over real-worlld networks

\begin{tabular}{|c|c|c|c|c|c|c|c|c|c|c|c|c|c|}
\hline \multicolumn{2}{|r|}{$\begin{array}{l}\text { Data } \\
\text { sets }\end{array}$} & \multicolumn{3}{|c|}{ Log-normal } & \multicolumn{3}{|c|}{ Poisson } & \multicolumn{3}{|c|}{$\begin{array}{l}\text { Power-law } \\
\text { Cutoff }\end{array}$} & \multicolumn{3}{|c|}{ Exponential } \\
\hline & & RMSE & KLD & MAE & RMSE & KLD & MAE & RMSE & KLD & MAE & RMSE & KLD & MAE \\
\hline \multirow{11}{*}{$\begin{array}{c}\text { Social } \\
\text { Networks }\end{array}$} & ego-Twitter(In) & 53.863 & 0.0169 & 2.9494 & 410.93 & 10.452 & 36.645 & 68.004 & 0.0397 & 4.1974 & 157.98 & 0.2733 & 11.567 \\
\hline & ego-Gplus(In) & 10.155 & 0.0678 & 0.2523 & 95.967 & 25.317 & 3.0371 & 30.925 & 0.1475 & 0.6821 & 50.098 & 1.3131 & 1.8328 \\
\hline & soc-Slashdot & 237.63 & 0.1058 & 10.549 & 684.36 & 10.069 & 42.407 & 19.598 & 0.0075 & 1.3599 & 434.25 & 0.6381 & 22.275 \\
\hline & soc-Delicious(In) & 281.34 & 0.0579 & 10.781 & 957.82 & 6.8432 & 56.634 & 66.896 & 0.0185 & 4.2366 & 535.11 & 0.4626 & 25.304 \\
\hline & soc-Digg(In) & 69.438 & 0.0552 & 1.9087 & 323.59 & 21.541 & 15.134 & 65.713 & 0.0441 & 1.9042 & 204.50 & 0.8907 & 8.0015 \\
\hline & soc-Academia & 91.003 & 0.0169 & 2.0921 & 542.38 & 7.2349 & 22.153 & 62.376 & 0.0255 & 1.9845 & 198.11 & 0.1924 & 6.6739 \\
\hline & LiveJournal(In) & 3473.6 & 0.0355 & 70.64 & $13.61 \mathrm{~K}$ & 9.1120 & 481.38 & 808.79 & 0.0101 & 24.501 & 7017.9 & 0.3449 & 186.01 \\
\hline & Dogster-Friendship & 42.539 & 0.0272 & 1.1459 & 494.49 & 14.575 & 15.309 & 182.47 & 0.1862 & 4.1579 & 165.19 & 0.4765 & 5.4623 \\
\hline & Higgs-Twitter(In) & 41.955 & 0.0134 & 0.5995 & 448.91 & 16.309 & 14.753 & 118.23 & 0.0914 & 2.8051 & 134.68 & 0.3163 & 4.4689 \\
\hline & Artist-Facebook & 24.071 & 0.0154 & 3.026 & 323.46 & 12.537 & 53.920 & 56.801 & 0.0458 & 6.6452 & 88.351 & 0.1799 & 13.623 \\
\hline & Athletes-Facebook & 15.461 & 0.0127 & 2.5674 & 175.95 & 4.7428 & 35.815 & 25.099 & 0.0324 & 4.3180 & 28.388 & 0.0586 & 6.2610 \\
\hline \multirow{4}{*}{$\begin{array}{l}\text { Citation } \\
\text { Networks }\end{array}$} & cit-HepTh(In) & 22.59 & 0.0255 & 2.331 & 153.39 & 8.0679 & 13.774 & 25.42 & 0.0464 & 2.816 & 58.74 & 0.2778 & 4.5286 \\
\hline & cit-HepPh(In) & 44.951 & 0.0189 & 4.4405 & 303.32 & 7.9234 & 46.775 & 36.887 & 0.0221 & 4.5287 & 107.32 & 0.1801 & 14.145 \\
\hline & cit-Patents(In) & 9612.7 & 0.0192 & 725.71 & $38.2 \mathrm{~K}$ & $\begin{array}{l}1.6549 \\
1.6549\end{array}$ & 3657.1 & 2424.5 & 0.0061 & 271.89 & $13.2 \mathrm{~K}$ & 0.0659 & 1147.5 \\
\hline & cit-Citeseer(In) & 353.26 & 0.0299 & 21.921 & 1507.6 & 2.566 & 109.15 & 195.67 & 0.0131 & 13.877 & 629.02 & 0.1486 & 44.301 \\
\hline \multirow{5}{*}{$\begin{array}{l}\text { Collaboration } \\
\text { Networks }\end{array}$} & ca-CondMat & 42.665 & 0.0082 & 6.5746 & 378.65 & 2.7263 & 80.781 & 62.929 & 0.0287 & 13.362 & 64.985 & 0.0472 & 16.873 \\
\hline & ca-AstroPh & 28.209 & 0.0312 & 6.8565 & 229.81 & 9.8703 & 55.51 & 50.604 & 0.0384 & 7.4579 & 68.185 & 0.1235 & 14.361 \\
\hline & ca-GrQc & 30.184 & 0.0515 & 12.148 & 193.94 & 2.3256 & 63.61 & 58.305 & 0.0659 & 18.259 & 69.169 & 0.1418 & 25.759 \\
\hline & ca-HepPh & 29.993 & 0.1011 & 6.8958 & 185.48 & 11.609 & 39.673 & 50.717 & 0.1128 & 8.2477 & 89.936 & 0.5187 & 17.589 \\
\hline & ca-HepTh & 55.618 & 0.0178 & 21.032 & 370.15 & 1.5051 & 133.68 & 89.425 & 0.0245 & 27.613 & 109.96 & 0.0551 & 43.882 \\
\hline \multirow{5}{*}{$\begin{array}{c}\text { Web } \\
\text { Graphs }\end{array}$} & Google(In) & 1514.5 & 0.0878 & 40.067 & 4442.6 & 4.712 & 154.92 & 188.01 & 0.0157 & 9.6549 & 2589.4 & 0.4419 & 76.441 \\
\hline & BerkStan(In) & 185.04 & 0.1002 & 2.0198 & 993.01 & 7.0379 & 11.9628 & 322.63 & 0.1037 & 2.8203 & 595.53 & 0.7438 & 6.6185 \\
\hline & Wikipedia2009(In) & 2720.9 & 0.0798 & 116.43 & 8425.7 & 3.6475 & 398.72 & 781.87 & 0.0082 & 35.531 & 4727.1 & 0.3431 & 213.66 \\
\hline & WikipediaLinkFr(In) & 240.18 & 0.0543 & 0.5234 & 762.72 & 25.726 & 4.0278 & 121.31 & 0.0622 & 0.5006 & 534.61 & 1.0217 & 2.0471 \\
\hline & Hudong(In) & 746.47 & 0.1593 & 6.5493 & 1975.73 & 11.088 & 25.837 & 75.362 & 0.0063 & 0.8323 & 1494.8 & 1.1798 & 15.836 \\
\hline \multirow{5}{*}{$\begin{array}{l}\text { Biological } \\
\text { Networks }\end{array}$} & Yeast-PPIN & 29.928 & 0.0496 & 9.3869 & 109.62 & 2.5149 & 39.181 & 4.9595 & 0.0175 & 2.9178 & 45.462 & 0.1234 & 13.786 \\
\hline & Diseasome & 23.282 & 0.1552 & 4.8906 & 55.985 & 3.0101 & 12.001 & 9.3332 & 0.0822 & 2.8587 & 31.709 & 0.2979 & 5.7013 \\
\hline & Bio-Mouse-Gene & 17.199 & 0.1878 & 2.5372 & 101.23 & 15.318 & 10.254 & 9.277 & 0.0943 & 1.6036 & 31.649 & 0.4882 & 3.6376 \\
\hline & Bio-Dmela & 46.271 & 0.0426 & 9.2857 & 206.44 & 3.6221 & 45.991 & 24.091 & 0.0162 & 5.0541 & 86.659 & 0.1724 & 18.048 \\
\hline & Bio-WormNet-v3 & 17.726 & 0.0851 & 3.9352 & 104.46 & 18.563 & 21.927 & 6.7764 & 0.0419 & 2.2424 & 40.795 & 0.3082 & 7.0826 \\
\hline \multirow{3}{*}{$\begin{array}{c}\text { Product } \\
\text { co-purchasing } \\
\text { networks }\end{array}$} & amazon0601(In) & 286.61 & 0.0102 & 16.881 & 2064.6 & 2.7267 & 140.46 & 297.39 & 0.0382 & 22.199 & 308.32 & 0.0574 & 24.114 \\
\hline & amazon0505(In) & 358.59 & 0.0125 & 19.123 & 2172.5 & 3.0551 & 144.34 & 260.85 & 0.0342 & 20.136 & 390.13 & 0.0628 & 26.178 \\
\hline & amazon0312(In) & 338.03 & 0.0116 & 17.742 & 2131.9 & 2.6839 & 140.75 & 273.39 & 0.0352 & 20.381 & 383.82 & 0.0639 & 26.299 \\
\hline \multirow{4}{*}{$\begin{array}{l}\text { Temporal } \\
\text { Networks }\end{array}$} & sx-mathoverflow(In) & 41.934 & 0.0634 & 5.3161 & 281.78 & 8.0773 & 32.868 & 92.603 & 0.0861 & 7.9912 & 129.69 & 0.4636 & 15.172 \\
\hline & sx-stackoverflow(In) & 341.96 & 0.0286 & 4.4267 & 2469.6 & 18.054 & 42.111 & 740.18 & 0.0685 & 7.2275 & 1324.4 & 0.6829 & 19.362 \\
\hline & sx-superuser(In) & 243.42 & 0.0616 & 13.199 & 1246.2 & 3.8103 & 68.010 & 354.72 & 0.0570 & 16.613 & 609.40 & 0.3891 & 34.655 \\
\hline & sx-askubuntu(In) & 212.91 & 0.0649 & 12.451 & 1228.7 & 2.6253 & 68.973 & 389.14 & 0.0719 & 20.113 & 555.44 & 0.3433 & 33.693 \\
\hline \multirow{3}{*}{$\begin{array}{l}\text { Communication } \\
\text { Networks }\end{array}$} & Email-Enron & 121.47 & 0.0873 & 8.445 & 426.39 & 6.8601 & 38.373 & 95.468 & 0.0689 & 7.664 & 230.41 & 0.5405 & 18.139 \\
\hline & Wiki-Talk(In) & 7978.6 & 0.1902 & 246.26 & $21.9 \mathrm{~K}$ & 1.2506 & 646.54 & 672.32 & 0.0036 & 25.905 & $16.5 \mathrm{~K}$ & 0.4879 & 542.31 \\
\hline & Rec-Libimseti(In) & 87.472 & 0.0755 & 1.4021 & 281.18 & 30.222 & 8.0019 & 28.059 & 0.0359 & 0.6971 & 166.18 & 0.8547 & 3.9402 \\
\hline \multirow{5}{*}{$\begin{array}{l}\text { Ground-truth } \\
\text { Networks }\end{array}$} & Wiki-Topcats & 272.99 & 0.0464 & 1.5159 & 1477.2 & 8.7468 & 12.121 & 389.86 & 0.0629 & 2.2289 & 832.23 & 0.6767 & 5.8936 \\
\hline & com-Friendster & $101 \mathrm{~K}$ & $\begin{array}{l}0.0404 \\
0.0762\end{array}$ & 4022.8 & $280 \mathrm{~K}$ & $\begin{array}{l}0.7408 \\
24.658\end{array}$ & $22.1 \mathrm{~K}$ & $\begin{array}{l}309.80 \\
17.8 \mathrm{~K}\end{array}$ & 0.0052 & 1025.5 & $193 \mathrm{~K}$ & 0.7216 & $\begin{array}{l}0.0930 \\
10.1 \mathrm{~K}\end{array}$ \\
\hline & com-LiveJournal & 2629.9 & 0.0299 & 51.656 & $10.9 \mathrm{~K}$ & 9.5778 & 401.89 & 497.89 & 0.0104 & 18.559 & 5230.3 & 0.2889 & 139.74 \\
\hline & com-Orkut & 452.92 & 0.0459 & 19.624 & 3118.1 & 11.839 & 135.17 & 261.75 & 0.0479 & 16.197 & 228.83 & 0.0599 & 14.496 \\
\hline & com-Youtube & 1422.2 & 0.1416 & 17.219 & 3838.9 & 3.4522 & 51.118 & 143.79 & 0.0045 & 2.2564 & 2515.4 & 0.6241 & 31.101 \\
\hline \multirow{5}{*}{$\begin{array}{c}\text { Brain } \\
\text { Networks }\end{array}$} & Human25890-session1 & 27.703 & 0.0222 & 4.1822 & 472.32 & 16.412 & 52.714 & 65.972 & 0.0509 & 7.8146 & 92.649 & 0.2272 & 15.977 \\
\hline & Human25890-session2 & 78.483 & 0.0471 & 14.289 & 1326.6 & 16.151 & 112.92 & 83.707 & 0.0162 & 6.709 & 183.06 & 0.1701 & 26.302 \\
\hline & Human25864-session2 & 83.489 & 0.0495 & 18.215 & 1433.3 & 17.298 & 143.91 & 106.20 & 0.0156 & 9.8828 & 212.96 & 0.1605 & 33.272 \\
\hline & Human25913-session2 & 99.615 & 0.0292 & 15.796 & 1614.4 & 18.899 & 171.16 & 223.39 & 0.0219 & 15.122 & 440.58 & 0.3331 & 59.629 \\
\hline & Human25886-session1 & 89.805 & 0.0568 & 20.819 & 1568.3 & 13.481 & 153.03 & 102.34 & 0.0154 & 11.296 & 207.52 & 0.1354 & 33.287 \\
\hline
\end{tabular}
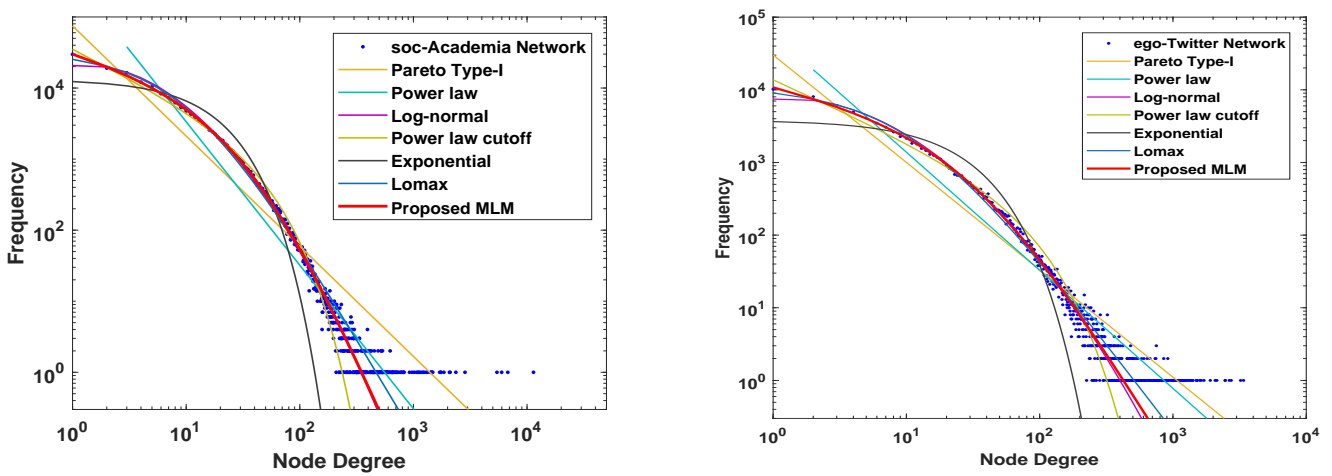

Figure 3: Degree distribution of soc-Academia and ego-Twitter networks in log-log scale 

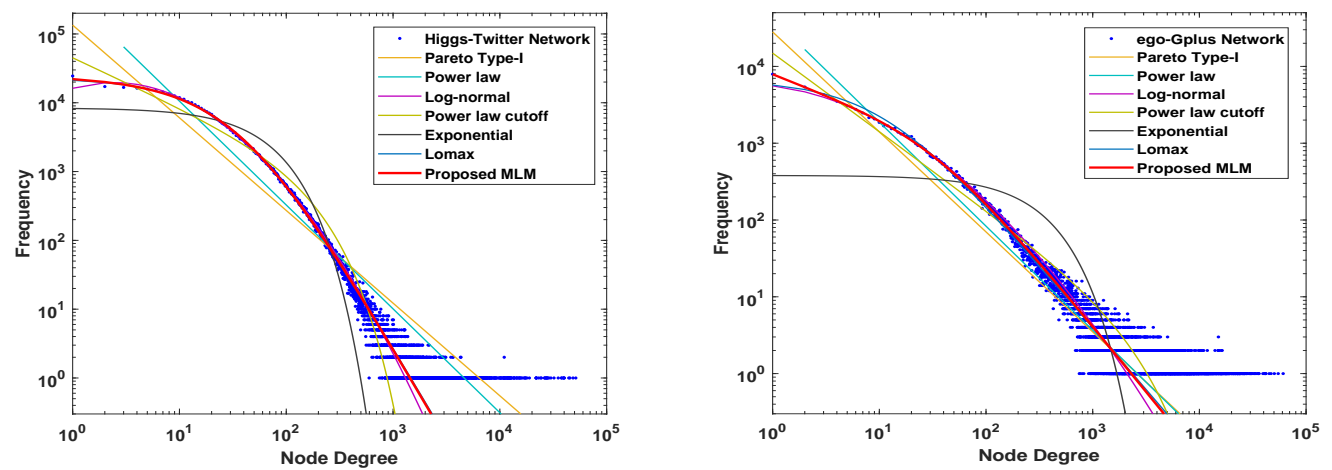

Figure 4: Degree distribution of Higgs-Twitter and ego-Gplus networks in log-log scale
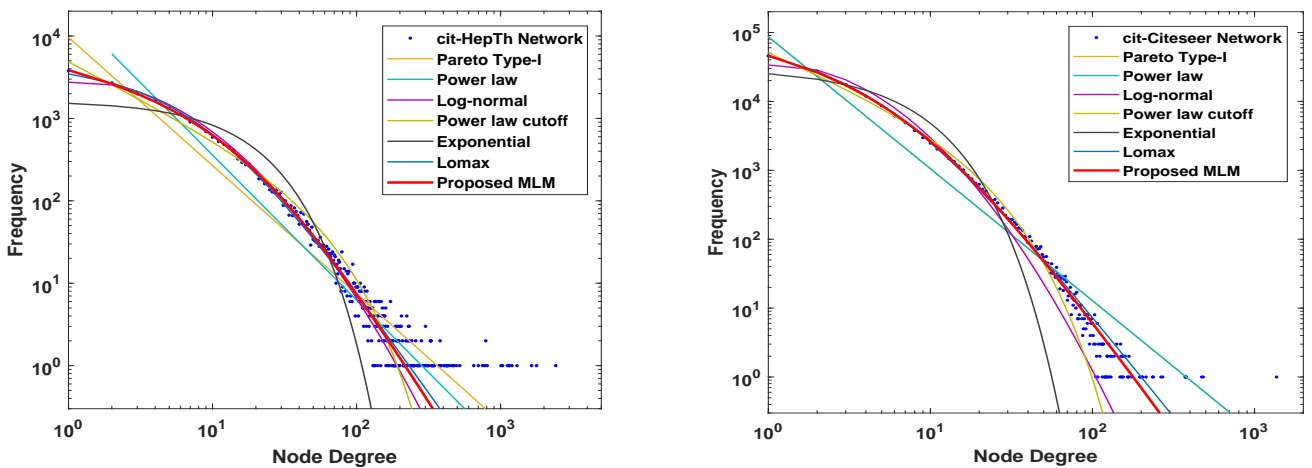

Figure 5: Degree distribution of cit-HepTh and cit-Citeseer networks in log-log scale
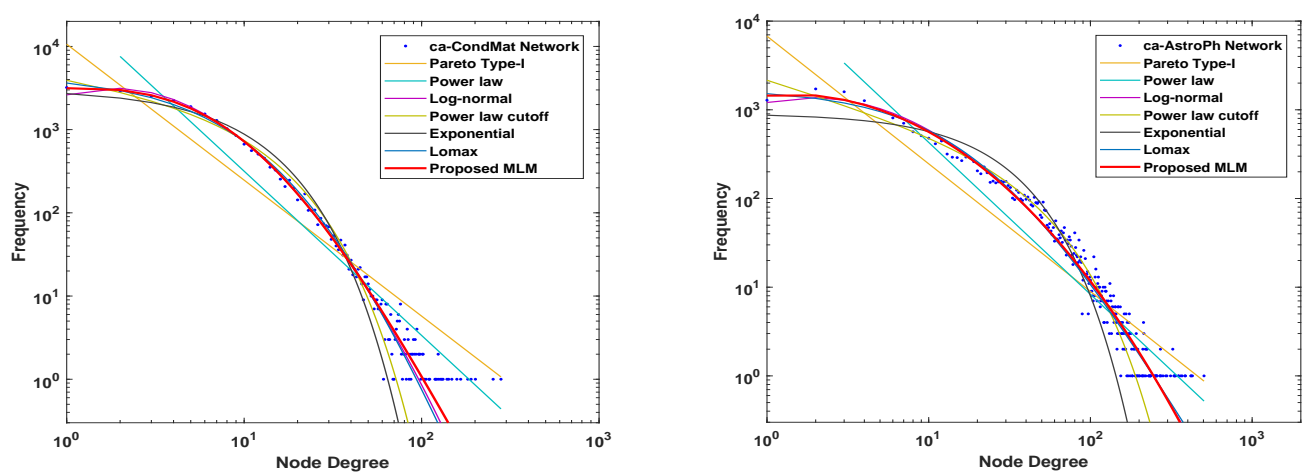

Figure 6: Degree distribution of ca-CondMat and ca-AstroPh networks in log-log scale 

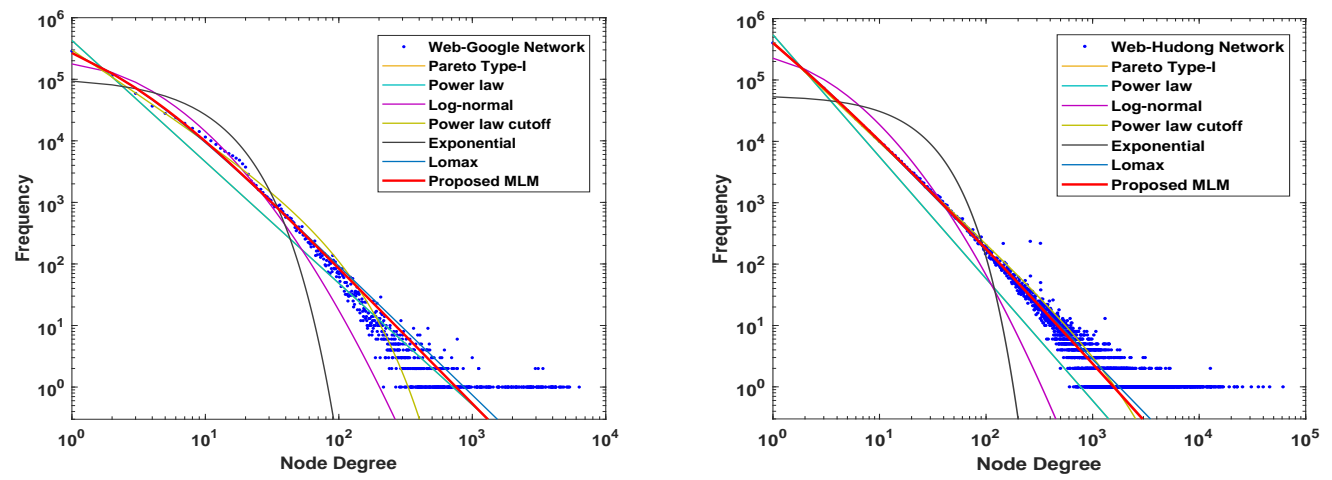

Figure 7: Degree distribution of Web-Google and Web-Hudong networks in log-log scale
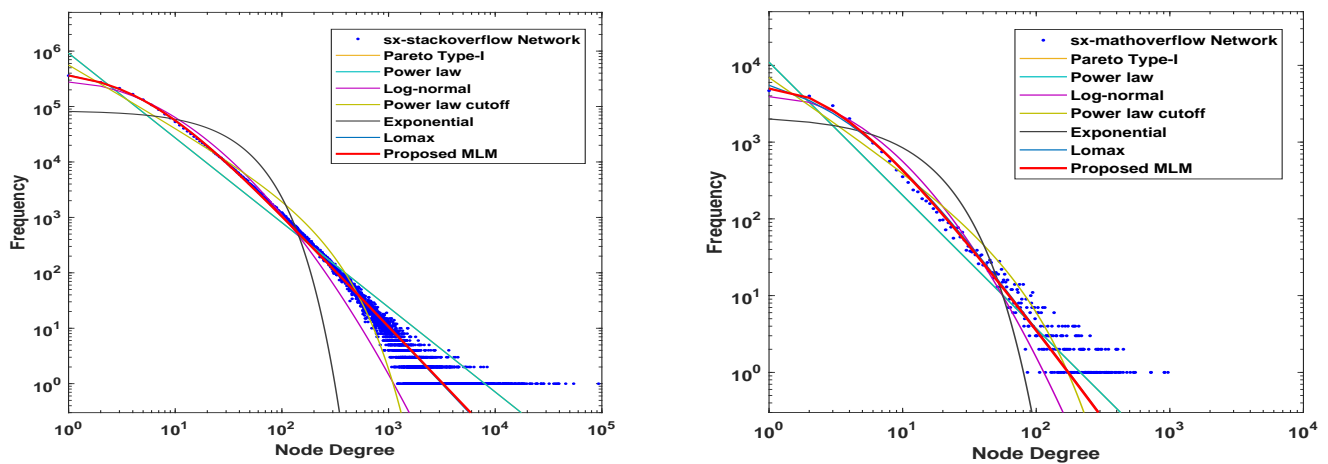

Figure 8: Degree distribution of sx-stack overflow and sx-mathoverflow networks in log-log scale
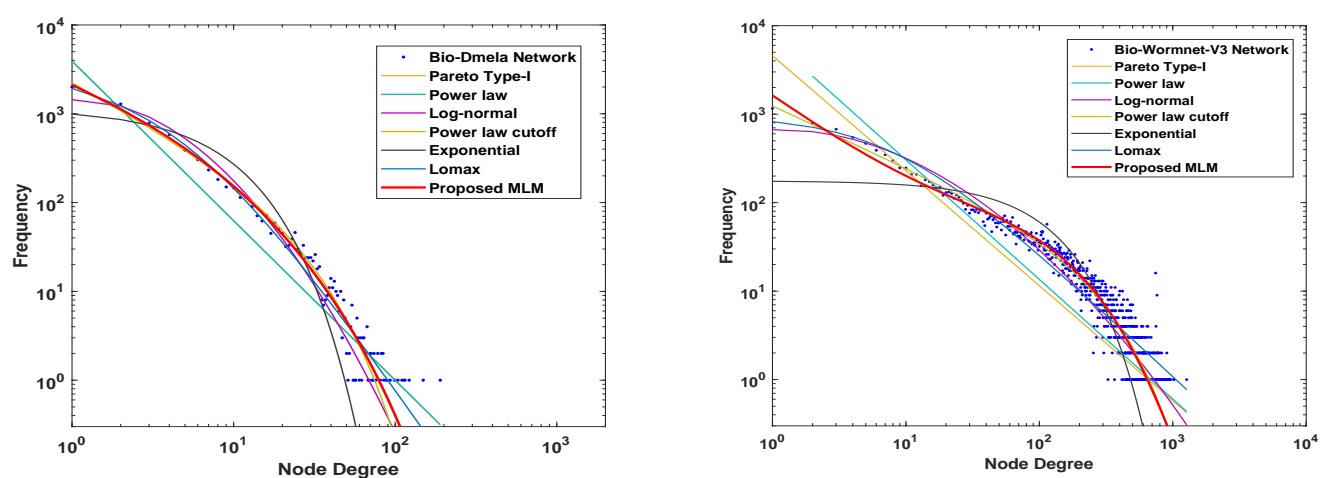

Figure 9: Degree distribution of Bio-Dmela and Bio-Wormnet-V3 networks in log-log scale 

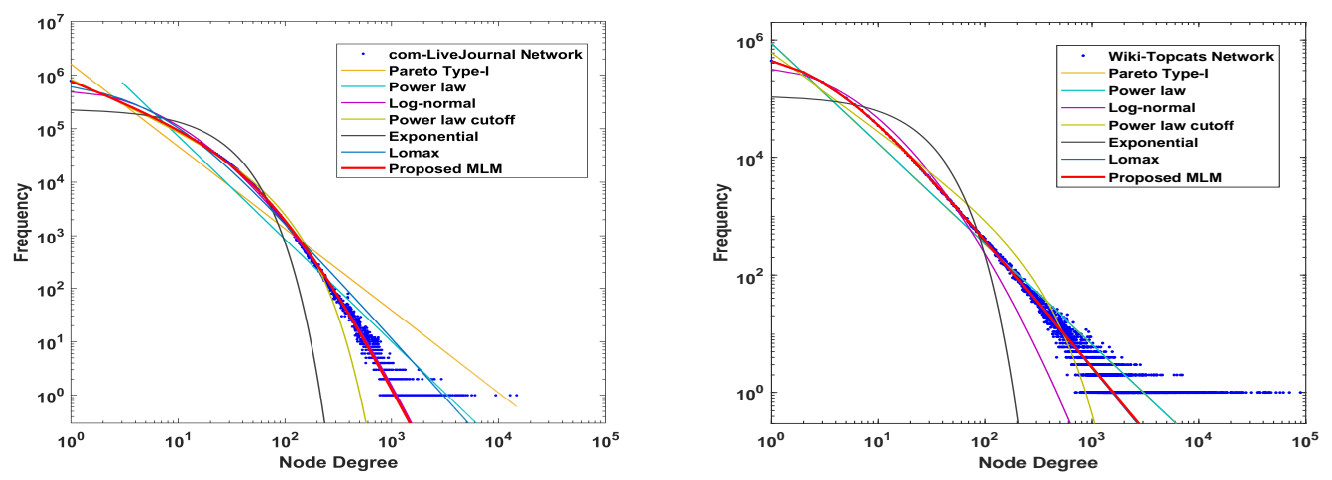

Figure 10: Degree distribution of LiveJournal and Wiki-Topcats networks in log-log scale

The effectiveness of the proposed MLM distribution can also be verified through the plotting of the fitted results of competitive distributions. For this purpose, the log-log plots of the of the original frequency distribution, the estimated frequency by MLM distribution and the frequency estimated by power-law, pareto, log-normal, power-law cutoff and exponential distributions are drawn for all the networks under consideration. Twenty four such examples have been provided in Figures 3, 10, These are the soc-Academia network, ego-Twitter network, Higgs-Twitter network, ego-Gplus network, cit-HepTh network, cit-Citeseer network, ca-CondMat network, ca-AstroPh network, Web-Google network, web-Hudong network, sx-stackoverflow, sx-mathoverflow, Bio-Dmela network, Bio-Wormnet-V3 network, com-LiveJournal and- com-Wiki-Topcats network. Few more plotted results are also provided in the supplementary section. We have omitted the plot of the poisson distribution due to its poor performances over all the networks. It is visually clear From Figures 3, 10 that the proposed MLM distribution provides better fit compared to the other competitive distributions in almost all of the networks since the proposed curve always passes through the middle of the scatter plot of the observed distribution. In a few cases the power-law cutoff and log-normal provide a better fit than the proposed distribution. It is visually clear from observing the social, biological, brain and citation networks that the entire node degree distribution can be better represented by the MLM distribution compared to other heavy tailed distributions. Thus the proposed MLM distribution, a modification of the Lomax distribution with non linear exponent in the shape parameter, can be used for effective and efficient modeling of the entire degree distribution of real-worlld networks without ignoring the lower degree nodes. The proposed MLM distribution provides more flexibility in the degree distribution modeling since the non-negative shape parameter are assumed to be expressed as a nonlinear function of the data. Empirical results also suggests the effectiveness of the proposed MLM distribution compared to others as depicted through Tables 1, 3] and Figures 3, 10.

\section{Conclusion and Discussion}

In this article, we have proposed a modified Lomax (MLM) distribution derived from a hierarchical family of Lomax distributions for flexible and efficient modeling of the entire node degree distribution of real-world complex networks. The proposed MLM distribution can be thought of as a generalization of the Lomax distribution with the nonlinear exponent in the shape parameter. We have theoretically established that the MLM distribution is heavy-tailed and right-tailed equivalent to the power-law distribution. Furthermore, we have shown a sufficient condition for the existence of the MLE for the parameters of MLM distribution using the notion of CV. The proposed MLM distribution can find MLE for the parameters at finite points when the value of $\mathrm{CV}>1$. We also theoretically justified that the MLM distribution is a function with regularly varying tails which belongs to the Maximum domain of attraction of the Frechet distribution. We have further studied the asymptotic behaviors of the MLM distribution in this context.

The proposed MLM distribution captures the heavy-tailed and nonlinear behavior of the entire degree distributions of real-world networks in the original and the log-log scale more adroitly. It also enables us 
to accurately characterize the degree distribution pattern which may have a significant impact on analyzing real-world networks in terms of their social or biological aspects, as the case may be. We have applied the proposed MLM distribution in modeling the entire degree distribution over 50 different real-world empirical data sets taken from diverse fields. Empirical results suggest that as compared to the power-law distribution or any other well-known distribution, our proposed MLM distribution produces a lower fitting error in terms of three statistical tests, viz. RMSE, KL-divergence, and MAE. We also demonstrated the statistical significance of the estimated MLM distribution with the help of the bootstrap Chi-square value. This generalization of the Lomax distribution by adding an additional parameter in the base model results in flexible modeling to the entire degree distribution of a real-world network compared to other heavy-tailed distributions unlike power-law. The proposed fit distribution sometimes helps us in better characterization of the evolution process of large scale real-world networks instead of explicitly performing the empirical study at each time step. Thus, by simulating the parameters of a proposed fit MLM distribution, one can easily capture the spatial structure and dynamical pattern of a real-world network as the network evolves over time. The dynamic pattern analysis of such structural properties in real-world networks is one of the future scopes of research.

\section{Acknowledgement}

The authors gratefully acknowledge the financial assistance received from Indian Statistical Institute (I. S. I.) and Visvesvaraya PhD Scheme awarded by the Government of India.

\section{References}

[1] R. Albert, A.-L. Barabási, Statistical mechanics of complex networks, Reviews of modern physics 74 (1) (2002) 47.

[2] R. Albert, H. Jeong, A.-L. Barabási, Error and attack tolerance of complex networks, nature 406 (6794) (2000) $378-382$.

[3] M. E. Newman, The structure of scientific collaboration networks, Proceedings of the national academy of sciences 98 (2) (2001) 404-409.

[4] M. E. Newman, The structure and function of complex networks, SIAM review 45 (2) (2003) $167-256$.

[5] A. Chacoma, G. Mato, M. N. Kuperman, Dynamical and topological aspects of consensus formation in complex networks, Physica A: Statistical Mechanics and its Applications 495 (2018) 152-161.

[6] F. D. Zarandi, M. K. Rafsanjani, Community detection in complex networks using structural similarity, Physica A: Statistical Mechanics and its Applications 503 (2018) 882-891.

[7] Y. Cui, X. Wang, J. Eustace, Detecting community structure via the maximal sub-graphs and belonging degrees in complex networks, Physica A: Statistical Mechanics and its Applications 416 (2014) 198-207.

[8] T. Nie, Z. Guo, K. Zhao, Z.-M. Lu, The dynamic correlation between degree and betweenness of complex network under attack, Physica A: Statistical Mechanics and Its Applications 457 (2016) 129-137.

[9] H. Shakibian, N. M. Charkari, Statistical similarity measures for link prediction in heterogeneous complex networks, Physica A: Statistical Mechanics and its Applications 501 (2018) 248-263.

[10] L. Muchnik, S. Pei, L. C. Parra, S. D. Reis, J. S. Andrade Jr, S. Havlin, H. A. Makse, Origins of power-law degree distribution in the heterogeneity of human activity in social networks, Scientific reports 3 (1) (2013) 1-8.

[11] A.-L. Barabási, R. Albert, Emergence of scaling in random networks, science 286 (5439) (1999) 509-512.

[12] R. Albert, H. Jeong, A.-L. Barabási, Diameter of the world-wide web, nature 401 (6749) (1999) $130-131$.

[13] A. Clauset, C. R. Shalizi, M. E. Newman, Power-law distributions in empirical data, SIAM review 51 (4) (2009) $661-703$.

[14] A.-L. Barabasi, The origin of bursts and heavy tails in human dynamics, Nature 435 (7039) (2005) $207-211$.

[15] F. Liljeros, C. R. Edling, L. A. N. Amaral, H. E. Stanley, Y. Åberg, The web of human sexual contacts, Nature 411 (6840) (2001) 907-908.

[16] J. H. Jones, M. S. Handcock, Sexual contacts and epidemic thresholds, Nature 423 (6940) (2003) 605-606.

[17] M. E. Newman, Power laws, pareto distributions and zipf's law, Contemporary physics 46 (5) (2005) 323-351.

[18] M. Seshadri, S. Machiraju, A. Sridharan, J. Bolot, C. Faloutsos, J. Leskove, Mobile call graphs: beyond power-law and lognormal distributions, in: Proceedings of the 14th ACM SIGKDD international conference on Knowledge discovery and data mining, 2008, pp. 596-604.

[19] A. D. Broido, A. Clauset, Scale-free networks are rare, Nature communications 10 (1) (2019) 1-10.

[20] P. Holme, Rare and everywhere: Perspectives on scale-free networks, Nature communications 10 (1) (2019) 1-3.

[21] I. Voitalov, P. van der Hoorn, R. van der Hofstad, D. Krioukov, Scale-free networks well done, Physical Review Research 1 (3) (2019) 033034.

[22] M. P. Stumpf, M. A. Porter, Critical truths about power laws, Science 335 (6069) (2012) 665-666

[23] A. Sala, H. Zheng, B. Y. Zhao, S. Gaito, G. P. Rossi, Brief announcement: revisiting the power-law degree distribution for social graph analysis, in: Proceedings of the 29th ACM SIGACT-SIGOPS symposium on Principles of distributed computing, 2010, pp. 400-401. 
[24] S. Chattopadhyay, A. K. Das, K. Ghosh, Finding patterns in the degree distribution of real-world complex networks: Going beyond power law, Pattern Analysis and Applications (2019) 1-20.

[25] S. Chattopadhyay, C. Murthy, S. K. Pal, Fitting truncated geometric distributions in large scale real world networks, Theoretical Computer Science 551 (2014) 22-38.

[26] K. Lomax, Business failures: Another example of the analysis of failure data, Journal of the American Statistical Association 49 (268) (1954) 847-852.

[27] M. Ahsanullah, Record values of the lomax distribution, Statistica Neerlandica 45 (1) (1991) $21-29$.

[28] A. S. Hassan, S. M. Assar, A. Shelbaia, Optimum step-stress accelerated life test plan for lomax distribution with an adaptive type-ii progressive hybrid censoring, Journal of Advances in Mathematics and Computer Science (2016) 1-19.

[29] A. Childs, N. Balakrishnan, M. Moshref, Order statistics from non-identical right-truncated lomax random variables with applications, Statistical Papers 42 (2) (2001) 187-206.

[30] S. Al-Awadhi, M. Ghitany, Statistical properties of poisson-lomax distribution and its application to repeated accidents data, Journal of Applied Statistical Science 10 (4) (2001) 365-372.

[31] N. Balakrishnan, M. Ahsanullah, Relations for single and product moments of record values from lomax distribution, Sankhyā: The Indian Journal of Statistics, Series B (1994) 140-146.

[32] K. Jayakumar, B. Krishnan, G. Hamedani, On a new generalization of pareto distribution and its applications, Communications in Statistics-Simulation and Computation 49 (5) (2020) 1264-1284.

[33] A. B. Atkinson, A. J. Harrison, Distribution of personal wealth in Britain, Cambridge Univ Pr, 1978.

[34] M. C. Bryson, Heavy-tailed distributions: properties and tests, Technometrics 16 (1) (1974) 61-68.

[35] M. Chahkandi, M. Ganjali, On some lifetime distributions with decreasing failure rate, Computational Statistics \& Data Analysis 53 (12) (2009) 4433-4440.

[36] A. S. Hassan, A. S. Al-Ghamdi, Optimum step stress accelerated life testing for lomax distribution, Journal of Applied Sciences Research 5 (12) (2009) 2153-2164.

[37] B. C. Arnold, Pareto distributions, Chapman and Hall/CRC, 2015.

[38] I. B. Abdul-Moniem, Recurrence relations for moments of lower generalized order statistics from exponentiated lomax distribution and its characterization, Journal of Mathematical and Computational Science 2 (4) (2012) 999-1011.

[39] M. Rajab, M. Aleem, T. Nawaz, M. Daniyal, On five parameter beta lomax distribution, Journal of Statistics 20 (1)

[40] A. El-Bassiouny, N. Abdo, H. Shahen, Exponential lomax distribution, International Journal of Computer Applications 121 (13).

[41] G. M. Cordeiro, E. M. Ortega, B. V. Popović, The gamma-lomax distribution, Journal of Statistical computation and Simulation 85 (2) (2015) 305-319.

[42] M. Tahir, M. A. Hussain, G. M. Cordeiro, G. Hamedani, M. Mansoor, M. Zubair, The gumbel-lomax distribution: properties and applications, Journal of Statistical Theory and Applications 15 (1) (2016) 61-79.

[43] D. E. Giles, H. Feng, R. T. Godwin, On the bias of the maximum likelihood estimator for the two-parameter lomax distribution, Communications in Statistics-Theory and Methods 42 (11) (2013) 1934-1950.

[44] P. Embrechts, C. Klüppelberg, T. Mikosch, Modelling extremal events: for insurance and finance, Vol. 33, Springer Science \& Business Media, 2013.

[45] S. Foss, D. Korshunov, S. Zachary, et al., An introduction to heavy-tailed and subexponential distributions, Vol. 6, Springer, 2011.

[46] C. Klüppelberg, Subexponential distributions and integrated tails, Journal of Applied Probability 25 (1) (1988) $132-141$.

[47] J. Leskovec, A. Krevl, SNAP Datasets: Stanford large network dataset collection, http://snap.stanford.edu/data (Jun. 2014).

[48] R. Rossi, N. Ahmed, The network data repository with interactive graph analytics and visualization, in: Twenty-Ninth AAAI Conference on Artificial Intelligence, 2015. 